Courant Institute of

Mathematical Sciences

Magneto-Fluid Dynamics Division

\title{
MASTER
}

\section{The Motion of a Charged Particle In a Nearly Axisymmetric Magnetic Field}

Harold Weitzner

U.S. Department of Energy Report and

Air Force Office of Scientific

Research Report

Plasma Physics

November 1980

New York University 


\section{DISCLAIMER}

This report was prepared as an account of work sponsored by an agency of the United States Government. Neither the United States Government nor any agency Thereof, nor any of their employees, makes any warranty, express or implied, or assumes any legal liability or responsibility for the accuracy, completeness, or usefulness of any information, apparatus, product, or process disclosed, or represents that its use would not infringe privately owned rights. Reference herein to any specific commercial product, process, or service by trade name, trademark, manufacturer, or otherwise does not necessarily constitute or imply its endorsement, recommendation, or favoring by the United States Government or any agency thereof. The views and opinions of authors expressed herein do not necessarily state or reflect those of the United States Government or any agency thereof. 


\section{DISCLAIMER}

Portions of this document may be illegible in electronic image products. Images are produced from the best available original document. 


\title{
UNCLASS IF IED
}

New York University

Courant Institute of Mathematical sciences

Magneto-Fluid Dynamics Division

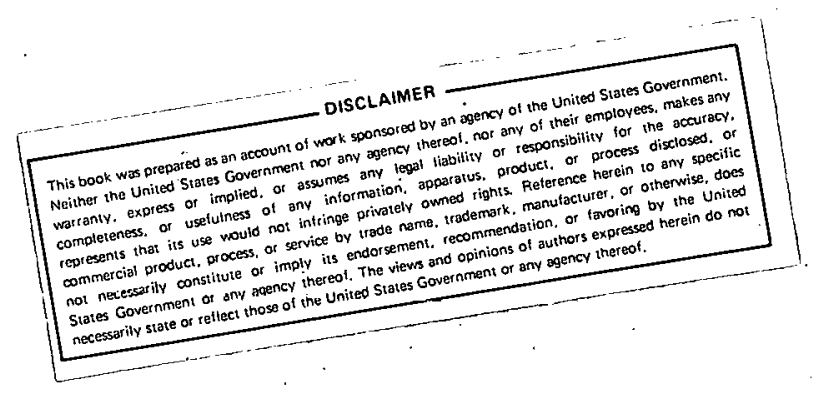

MF-97

$\mathrm{DOE} / \mathrm{ER} / 03077-169$

\author{
THE MOTION OF A CHARGED PARTICLE IN A \\ NEARLY AXISYMMETRIC MAGNETIC FIELD
}

Harold Weitzner

November 1980

U. S. Department of Energy

Contract No. DE-AC02-76ERO3077

and

Air Force Office of Scientific Research

Contract No. 76-2881

UNCLASSIFIED 


\author{
The Motion of a Charged Particle in a \\ Nearly Axisymmetric Magnetic Field \\ Harold Weitzner \\ Courant Institute of Mathematical Sciences \\ New York University \\ 251 Mercer Street \\ New York, N. Y. 10012
}

\begin{abstract}
The motion of a charged particle in a static magnetic field is studied by means of repeated canonical transformations of a Hamiltonian system. Adiabatic invariants are generated based on the assumption that the particle larmor radius is small compared with the characteristic distance over which the magnetic fleld varies. Unlike many earlier treatments the transformations presented here preserve the axisymmetry of the dynamics when the magnetic field is axisymmetric. It is assumed that the magnetic field consists of a small nonaxisymmetric part plus the axisymmetric toroidal and poloidal parts. After the introduction of the magnctic moment adiabatic invariant the motion of the guiding center is studied. The results depend sensitively on the ratio of the poloidal magnetic field to the total magnetic field. In some cases a second adiabatic invariant exists and direct inferences concerning long time particle drifts are possible. In one case where a second adiabatic invariant fails to exist, long term drifts are studied by conventional perturbation expansions. At some points resonance or lack of resonance phenomena appear and determine the drift effects.
\end{abstract}


I. Introduction

The motion of a single charged particle in a given magnetic field, one of the first problems studied in plasma physics, (1) has recently become of interest again. Two topics of immediate significance in magnetic fusion energy research have sparked the current concern: (2) the confinement of high energy alpha particles and the drift motion of uxderate energy particles induced hy small magnetic ficlds that bitak axial symmetry. The large body of older theory has been used to provide a base for extensive numerical computations. Here, we examine the problem with analytic tools to discover what improved descriptions of single particle motion we are able to provide. The critical concept In the study of the single particle dynamics is the existence of adiabatic invariants of the motion which allows great simplifications in the study of the dynamics. The adiabatic invariants are associaten with the presence of a physical parameter which is small, the ratio of the larmor radius to the characteristic distance over which magnetic fields vary; and we denote this parameter by $\varepsilon$. Unlike the earlier treatments we assume that parts of the axisymmetric magnetic field llay be small in $\varepsilon$ and we also take the symmetry breaking field to be small in e. Not ourprisingly, we find that these assumptions do allow considerably more analysis than is possible without them.

The older work has shown that provided $\varepsilon$ is small and both $B$ and $1 . / B$ are of order 1 in $\varepsilon$, whire $B$ is the given magnetic field, then there is a first adiabatic invariant, the magnetic moment of the particle. Using the approximate constancy of the magnetic moment, one may eliminate part of the single particle motion and study the simpler motion of the guiding centers of the particles. In many cases a second 
adiabatic invariant exists as well, and this second invariant, often called the longitudinal invariant, may be used to characterize the slow drifts of the guiding centers across the magnetic field lines. We are not concerned with the third or flux invariant, which also may exist in some cases.

The motion of a single particle is given by Hamilton's equations with the standard Hamiltonian for a single charged particle in a given magnetic field. C. S. Gardner(3) has applied the particularly. powerful and elegant procedure of repeated canonical transformations to this problem. This technique gives a systematic perturbation procedure.. which preserves the Hamiltonian character of the system and allows the elimination of degrees of freedom as the adiabatic invariants are introduced. The methods allow the isolation of drift motions and give reliable estimates of time intervals over which results apply. A major difficulty with the use of canonical transformations is that the great freedom of choice of transformations leaves the user unsure of how to start. After the first step, the process is fairly systematic, but that first step is far from obvious or natural. As flrst carried out by Gardner, the procedure had the unfortunate property that the axisymmetry of particle motion in axisymetric magnetic fields was destroyed. This difficulty has occurred in other treatments as well, (4) although there is an ad hoc device to eliminate the problem in lowest order. (5) One of our purposes is to present consistent perturbation schemes which preserve axisymmetric when present but allow for its absence. Our other intention is to examine drift motion in three distinct cases. We find the use of repeated canonical 
transformations ideally suited to our goals. Our treatment is based on Gardner's approach.

In order to discuss the drift motion problem we must describe the magnetic field more carefully. We treat the case of static magnetic fields with no electric field; time dependent magnetic fields or elertric ficldo are Leadily introduced tollowing Gardner's paradigm. We introduce cylindrical coordinates $(r, \theta, z)$ and we assims that the magnetic field $\underset{\sim}{B}$ is the sum of an axisymmetric magnetic field independent of $\theta$ and a nonaxisymmetric field $\mathrm{B}_{\mathrm{NA}} \cdot$ The axisymmetric field is itself the sum of a toroidal field $\hat{\theta} B_{\mathrm{T}}$ plus the poloidal field $B_{p}$ with components in the $\hat{r}$ and $\hat{z}$ directions only. The flux surfaces we mention subsequently are those of the axisymmetric field. We assume throughout that $\mathrm{B}_{\mathrm{NA}} / \mathrm{B} \sim \varepsilon$. We find that the drift motion depends critically on the ratio $B_{P} / B$. We consider separately the three cases $\mathrm{B}_{\mathrm{P}} / \mathrm{B} \sim 1, \mathrm{~B}_{\mathrm{P}} / \mathrm{B} \sim \sqrt{\varepsilon}$, and $\mathrm{B}_{\mathrm{P}} / \mathrm{B} \sim \varepsilon$. In earh case we obtain canonically conjugate coordinates and momenta which show that the motion is compounded of several motions on differing time scales. The gyro rolation of the particle about its guiding oenter is on the fastust time scale in each case. The introduction of the magnetic moment adiabatic invariant effectively eliminates this motion from" the Hamiltonian and drops the number of pairs of canonically conjugate variables from three to two. The reduced Hamiltonian describes the motion of the guiding center. We now consider the dymamics of the guiding center.

When $\mathrm{B}_{\mathrm{P}} / \mathrm{B} \sim 1$ there are two classes of guiding center orbits. The passing particles, or untrapped particles, encircle the magnetic axis while the trapped particles do not encircle the magnetic axis. The 
trapped particles possess a second adiabatic invariant, from whose existence we show that the quiding centers are bounded to lie near a given flux surface for very long times. Only nonadiabatic effects will allow significant drifts across flux surfaces. The description of the passing particles is delicate and our analysis does not give a full picture. For certain very special classes of magnetic fields a second adiabatic invariant exists and from its existence one may construct a Hamiltonian for the very slow particle drifts across flux surfaces. These drifts may indeed be large. The condition for these results is a particular approximate resonance in the motion. If the resonance is not present then no second adiabatic invariant exists and we are unable to describe the drifts easily. It becomes clear that in such rather general cases high order accuracy of the quiding oenter motion is needed to obtain the drifts correctly. This scaling is discussed in the third section.

When $\mathrm{B}_{\mathrm{P}} / \mathrm{B} \sim \varepsilon$ there is always a second adiabatic invariant. The dependence of the second adiabatic invariant on the canonical angular momentum $p_{0}$ and the relation between $p_{\theta}$ and the flux surface on which a particle lies are drastically different from the case just discussed. The arift orbits, even without nonaxisymmetric perturbing fields, are large and show large deviations from a given flux surface. The only significant effect of the nonaxisymetric fields is to rearrange the orbits near the critical points in the phase plane of the drift Hamiltonian. This scaling is treated in the fourth section.

The intermidiate scaling $B_{p} / B \sim \sqrt{ } \varepsilon$ is the most difficult of the three. With one set of canonical variables it seems that a second adiabatic invariant exists. However, it is shown that the time 
interval over which its constancy is valid is severely limited. While it might appear on the limited time interval that the invariant is constant, its value must gradually change. With another set of canonically conjugate variables no second adiabatic invariant can be constructed. However, the motion can be described further by means of a more or less conventional perturbation expansion. Aqain the orhits separate into trapped and untrapped particles. Thé Liduped particle drift across the flux surface por particle period is evaluated. Thus, the drift may be described as a discrete process. Summed over many periods resonance ooherence effects may enhance the drift, or lack of resonance may average the drifts to an effect of much smaller order of magnitude. We have estimated the smaller nonresonant drifts and append a few comments of a purely qualitative nature. The untrapper particle drifts are only briefly alluded to in order to indicate that small, nonresonant drifts will be present here too. The analysis of this scaling is presented in section five. In addition, an appendix contains many of an details of an canonical transformatinns to high enough order to obtain the guiding genter Hamiltonian.

The essential element that allows the preservation of axisymetry when appropriate is the introduction of particular parametrizations of the vector potential of the magnetic field. The usual treatments employ the so called $(\alpha, \beta, s)$ Clehsch variables, $\beta=\nabla \alpha \times \nabla ;$. In the second section we present the gauge transformation of the vector potential which generates the several coordinate functions we use.

It may be of use to point out the reader the origins of the complexity of the particle dynamics we have attempted to describe. There are two major, and distinct problems. The first problem relates 
to the question of when an adiabatic invariant exists. In order to have an adiabatic invariant one must have two distinct properties of the Hamiltonian. First, one must have the motion a compound of motions on different time scales, and second, one must have a family of approximating, periodic orbits on the fastest time scale. In some cases the family of approximating, periodic orbits may not exist and then no invariant exists. The other problem $r \in$ lates to the parametrization of the magnetic field and the selection of canonically conjugate variables. When one scales components of the magnetic field in the small parameter $\varepsilon$, the order of magnitude of canonical variables shifts too, and the order of magnitude of canonical variables affects the time scales on which variables change. Further, variables that are originally functionally independent may become, to leading order, functionally dependent. The implications of these effects could be seen in the older works; they just had not been examined with any thoroughness. 


\section{Kinematic Preliminaries}

In this section we introduce the appropriate gauge for the vector potential associated with a given magnetic field and the small larmor radius scalings for the various magnetic field scalings. We employ cylindrical coordinates $r, \theta, z$ throughout. Let $\tilde{A}$ be a be a vector potential for a given magnetic field $\mathbf{B}$,

$$
B=\stackrel{D}{\sim} \times \tilde{A} \text {. }
$$

We might equally weli set:

$$
B=D \times A^{\prime}
$$

where $\tilde{A}^{\prime}=\tilde{A}+\nabla \beta(r, \theta, z)+\nabla \gamma(r, z)$. We introduce the notation

$$
\left\langle f\left(r, \theta, z_{i}\right)\right\rangle=\frac{1}{2 \pi} \cdot \int_{0}^{2 \pi} f\left(r, \theta^{\prime}, z_{z}\right) d \theta^{\prime},
$$

and clearly $\langle f(x, \theta, z)\rangle$ is independent of $\theta$. We see that

$$
\begin{aligned}
A_{\theta}^{\prime}(r, \theta, z)= & \left.\tilde{A}_{\theta}(r, \theta, z)\right\rangle+\left(\tilde{A}_{\theta}(r, \theta, z)-\left\langle\tilde{A}_{\theta}(r, \theta, z)>\right)\right. \\
& +\beta, \theta(r, \theta, z) / r .
\end{aligned}
$$

so that if we set

$$
B(r, \theta, z)=r \oint d \theta^{\prime}\left(\left\langle\tilde{A}_{\theta}(r, \theta, z)\right\rangle-\tilde{A}_{\theta}\left(r, \theta^{\prime}, z\right)\right),
$$

where $\beta(r, \theta, z)$ is a periodic function of theta, and

$$
\psi(r, z)=\left\langle\tilde{A}_{\theta} \cdot(r ; \theta, z)\right\rangle / r,
$$

then 


$$
A_{\theta}^{\prime}(r, \theta, z)=\psi(r, z) / r \text {. }
$$

We now turn to the specification of $\gamma(r, z)$. We seek to find $\gamma(r, z)$ and $\lambda(r, z)$ such that

$$
\begin{aligned}
& \left\langle\tilde{A}_{r}\right\rangle+\langle B\rangle, r+\gamma, r(r, z)=\lambda(r, z) \psi, r(r, z) \\
& \left\langle\tilde{A}_{z}\right\rangle+\langle B\rangle, z+\gamma, z(r, z)=\lambda(r, z),{ }_{, z}(r, z) .
\end{aligned}
$$

A necessary and sufficient condition that such a $\gamma(x, z)$ exist is that

$$
\left\langle\tilde{A}_{r}\right\rangle, z-\left\langle\tilde{A}_{z}\right\rangle, r=\gamma, z^{\psi}, r-\lambda, r^{\psi}, z \text {, }
$$

or

$$
\langle B\rangle \cdot \nabla \lambda(r, z)=r\left\langle B_{\theta}(r, \theta, z)\right\rangle \text {. }
$$

We may use (4) to determine $\lambda(x, z)$ although $\lambda(r, z)$ will not, in general, be single-valued. With $\lambda(r, z)$ satisfying (4) and $\gamma(r, z)$ chosen consistently we have obtained the following representation of the vector potential

$$
A^{\prime}=\lambda(r, z) \nabla \psi(r, z)+\hat{\theta} \psi(r, z) / r+\hat{r} A_{r}(r, \theta, z)+\hat{z} A_{z}(r, \theta, z)
$$

where

$$
\left\langle A_{r}(r, A, z)\right\rangle=\left\langle A_{z}(r, \theta, z)\right\rangle=0 .
$$

We Identily the magnctic field generated by $\lambda(r, z)$ and by $\psi(r, z)$ as the axisymetric magnetic field, while $A_{r}(r, \theta, z)$ and $A_{z}(r, \theta, z)$ 
generate the nonaxisymmetric field. Thus, we find that the axisymetric toroidal field is

$$
B_{T}=\hat{\theta} \cdot\left(\lambda, z^{\psi}, r-\lambda, r^{\psi}, z\right)
$$

while the axisymetric poloidal field is

$$
B_{P}=-\hat{r} \psi, z / r+\hat{z} \psi, r / r \text {, }
$$

so Lhat we may interpret $\psi(r, z)$ as the usual flux function. Finally, lie nunaxisymetric tield has the representation

$$
B_{N A}=\hat{r} A_{z, \theta} / r+\hat{\theta}\left(A_{r, z}-A_{z, r}\right)-\hat{z} A_{r, \theta} / r .
$$

We shall make use of one further function, $S(r, z)$, introduced so that the curves $S(x, z)=$ const. are orthogonal to the curves $\psi(r, z)=$ const.

$$
0=\nabla \psi \cdot \nabla S=\psi, r^{S}, r+\psi, z^{S}, z \cdot
$$

We next irileculuce the smill larmor radius scaling. We adopt the convention that $B$ is $O(1)$ in the larmor radius parameter, and more precisely that both $B$ and $1 / B$ are $~(1)$; that is $B$ may not become small $m$ likigu. Since the small yalduter $\varepsilon$ is the ratio of the larmor radius to characteristic distance of magnetic field variation, we may take either the larmor radius as small, or the characteristic distance of magnetic field variation as large. It is, in fact, simpler to take the larmor radius itself as $O(1)$ ard the other parameter as large. Thus, if we have a tunction of space $f(x, y, z, \varepsilon)$, then if the distance of spatial variation were large, we would expect $f$ to have the representation $\mathrm{f}(\mathrm{X}, \mathrm{Y}, \mathrm{z}, \varepsilon)=\mathrm{F}(\varepsilon \mathrm{X}, \varepsilon \mathrm{Y}, \varepsilon \mathrm{Z}, \varepsilon)$ where $\mathrm{F}(\mathrm{X}, \mathrm{Y}, \mathrm{Z}, \mathrm{E})$ has a.l its derivatives of order 1 with respect to its arguments $X, Y, Z, E$. Under these assumptions $\nabla f=\varepsilon\left(F, \varepsilon x^{x}+F, \varepsilon y+\hat{y}+F, \varepsilon z^{z}\right)$ and we see that $f$ 
changes slowly in $x, y, z$. The analogous representation for a function in polar coordinates $g(x, \theta, z, \varepsilon)$ would be

$$
g(r, \theta, z, \varepsilon)=G(\varepsilon r, \theta, \varepsilon z, \varepsilon)
$$

where again $G(R, A, Z, E)$ would have all derivatives of order 1 with respect to the arguments. Now we find

$$
\nabla g=\varepsilon\left(G, \varepsilon r^{r}+G_{, \theta} \hat{\theta} /(\varepsilon r)+G_{,} \varepsilon z^{\hat{z}}\right.
$$

so that we require, in general that $l /(\varepsilon r)$ be $O(1)$ in order that $\nabla g$ be $O(\varepsilon)$. That is, we must assume that we are far enough from the axis of the cylindrical coordinate system that $\varepsilon r$ not be small. We know that near the axis quantities may vary rapidly so this restriction is not surprising or unreasonable. Physically, we must be many larmor radii from the axis.

We now give the scaled versions of the vector potential for the several cases. When $B_{\mathrm{T}} \sim 1$ or smaller, $B_{\mathrm{p}} \sim 1$ and $\mathrm{B}_{\mathrm{NA}} \sim \varepsilon$ we set

$$
\begin{aligned}
\Delta^{\prime}=\lambda(\varepsilon r, \varepsilon z)\left(\hat{r} \psi, \varepsilon r^{(\varepsilon r, \varepsilon z)+} \hat{z}_{\psi}, \varepsilon z^{(\varepsilon r, \varepsilon z)) / \varepsilon}\right. \\
+\hat{\theta} \psi(\varepsilon r, \varepsilon z) /\left(\varepsilon^{2} r\right)+A_{r}(\varepsilon r, \theta, \varepsilon z) \hat{r}+A_{z}(\varepsilon r, \theta, \varepsilon z) \hat{z}
\end{aligned}
$$

where again $\left\langle A_{r}(\varepsilon r, \theta, \varepsilon z)\right\rangle=\left\langle A_{Z}(\varepsilon r, \theta, \varepsilon z)\right\rangle=0$. We find

$$
\begin{aligned}
& B_{\mathrm{T}}=\hat{\theta}\left(\lambda, \varepsilon z^{\psi}, \varepsilon r-\lambda, \varepsilon r^{\psi}, \varepsilon z\right) \\
& B_{P}=-\hat{r} \psi, \varepsilon z /(\varepsilon r)+\hat{z} \psi, \varepsilon r /(\varepsilon r),
\end{aligned}
$$

and

$$
B_{N A}=\varepsilon\left\{\hat{r} A_{z, \theta} /(\varepsilon r)+\hat{\theta}\left(A_{r, \varepsilon z}-A_{z, \varepsilon r}\right)-\hat{z} A_{r, \theta} /(\varepsilon r)\right\}
$$

so that provided $(\varepsilon r)$ is $O(1)$ and not smaller $B_{\mathrm{T}}=O(1), B_{\mathrm{P}}=O(1)$ and $\mathrm{B}_{\mathrm{NA}}=O(\varepsilon)$. Thus, the factors of $\varepsilon$ in the denominators of (8) are 
needed to make $B$, which is a derivative of $A^{\prime \prime}, O(1)$ : and not smaller. If we multiply the $\theta$ component of (8) by $\varepsilon^{9}$ then the poloidal component of $B$ is multiplied by $\varepsilon q$. Thus, if we wish $B_{p} \sim \varepsilon\left(B_{p} \sim \sqrt{ } \varepsilon\right)$ then we multiply the $\theta$ component of $(8)$ by $\varepsilon(\gamma \varepsilon \varepsilon)$.

The Hamiltonian for the single: particle motion is

$$
H=(1 / 2)\left\{\left(P_{r}-A_{r}^{\prime}\right)^{2}+\left(D_{A} / x-A_{A}^{\prime}\right)^{2}+\left(P_{z}-A_{z}^{\prime}\right)^{2}\right\}
$$

and

$$
\begin{aligned}
& v_{r}=p_{r}-A_{r}^{v} \\
& v_{\theta}=p_{\theta} / r-A_{\theta}^{\prime} \\
& v_{z^{\prime}}=p_{z^{\prime}}-A_{z}^{v}
\end{aligned}
$$

Since we have taken $B \sim 1$ and the larmor radius $O(I)$ we conclude that $\mathrm{V} \sim \mathrm{I}$ as well. We are now ready to start the study of particle dynamies. 
III. The Guiding Center Expansion in the General Case

In the general magnetic field configuration we take $B_{P} \sim 1, B_{T} \leqslant 1$ while $B_{\mathrm{NA}} \sim \varepsilon$. Our results correspond to the generally expected picture of single particle motion. After an appropriate symmetry preserving canonical transformation we show that the dynamics is composed of motions on three distinct time scales. The fastest motion is the gyro rotation of the particle about its guiding center. The introduction of the magnetic moment adiabatic invariant by successive canonical transformations eliminates two degrees of freedom and the fastest time scale from the Hamiltonian motion. The next slower motion is the guiding center motion. On this time scale there are two classes of orbits, trappen (or banana) orbits for which the trajectory is essentially bounded and periodic in the appropriate coordinate, and untrapped orbits which may or may not be periodic in the appropriate coordinates. In either case the motion on this time scale is essentially on a given flux surface. In the case of axisymmetry the slowest time scale is largely irrelevant to the motion and the preceding description is complete with particles elfectively constrained to be near a given flux surface. Small, nonaxisymmetric perturbations raise the possibility of significant particle drifts on the slowest time scale. We show that for the trapped particles a second adiabatic invariant exists and that its existence allows the elimination of two more degrees of freedom from the Hamiltonian. Nevertheless, we show that on the slowest time scale particles are effertively confined to a given flux surface. For the untrapped particles a second invariant may or may not exist depending on the existence or nonexistence of resonances between two modes of motion. 
The effects of resonances are explored, albeit not conclusively. We see that it is possible for an adiabatic invariant to exist leading to a Hamiltonian on the slowest time scale with large particle drifts. Equally well the drifts could be small, or even no second adiabatic invariant might exist. The origin of the complexity is quite simple. In order that an adiabatic invariant exist it is necessary not ony that there be a separation of time scales but also that. on the faster coalc the motion must be periodic. We have the separation of time scales but we may not have periodic orbits.

We may readily construct the Hamiltonian for the motion from (8) and $(12)$ as

$$
\begin{aligned}
\cdot H=(1 / 2) \mid\left(P_{r}-\lambda \psi, \varepsilon r / \varepsilon-A_{r}\right)^{2} & -\left(P_{z}-\lambda \psi, \varepsilon z\right. \\
& \left.+\varepsilon-A_{z}\right)^{2} \\
& \left.+\left(\varepsilon P_{\theta}-\psi / \varepsilon\right)^{2} /(\varepsilon r)^{2}\right\}
\end{aligned}
$$

We consider $\varepsilon r$ and $\varepsilon z$ as functions of $\psi$ and $S$ so that $\lambda(\varepsilon r, \varepsilon z)$, $\psi, \varepsilon r^{(\varepsilon r, \varepsilon z)}$ and $\psi, \varepsilon z(\varepsilon r, \varepsilon z)$ are functions of $\psi$ and $S$ or $\lambda=\lambda(\psi, S)$, etc. We introduce the generator of a transformation $F\left(p_{1}, P_{2}, P_{3}, r, \theta, z\right)$ involving the old coordinates $r, A, z$ and new momenta $p_{1}, p_{2}, p_{3}$

$$
F=\left(\psi / \varepsilon-c p_{1}\right)\left(p_{3}+\lambda / \varepsilon\right)+S p_{2} / \varepsilon+\theta p_{1}-\lambda, \psi\left(\psi / \varepsilon-\varepsilon p_{1}\right)^{2} / 2
$$

and from the relations

$$
\begin{gathered}
q_{i}=\frac{\partial F}{\partial p_{i}}, \quad i=1,2,3 \\
\left(p_{r}, p_{\theta}, p_{z}\right)=\left(\frac{\partial}{\partial r}, \frac{\partial}{\partial \theta}, \frac{\partial}{\partial z}\right) F
\end{gathered}
$$

we readily conclude 


$$
\begin{aligned}
& \psi=\varepsilon p_{1}^{2}+\varepsilon q_{3} \\
& s=\varepsilon q_{2} \\
& \theta=q_{1}+\lambda(\psi, s)+\varepsilon p_{3}-\varepsilon q_{3} \lambda, \psi(\psi, s)
\end{aligned}
$$

and

$$
\begin{aligned}
& p_{\theta}=p_{1} \\
& \left.P_{r}-\lambda \psi, \varepsilon r^{/ \varepsilon}=\psi, \varepsilon r^{\left(P_{3}-\varepsilon q_{3}^{2} \lambda, \psi \psi\right.} / 2\right) \\
& +s_{, \varepsilon r}\left(p_{2}+q_{3}^{\lambda}, s^{-} \varepsilon q_{3}^{2} \lambda, \psi S^{/ 2}\right) \\
& p_{z}-\lambda \psi, \varepsilon z / \varepsilon=\psi, \varepsilon z\left(p_{3}-\varepsilon q_{3}^{2} \lambda, \psi \psi / 2\right) \\
& +S_{, \in Z}\left(p_{2}+q_{3}^{\lambda}, S^{-\varepsilon} q_{3}^{2} \lambda, \psi S^{(2)}\right. \text {. }
\end{aligned}
$$

Following the ideas of Gardner we must now determine the magnitude (in $\varepsilon$ ) of the new canonical variables $\left(p_{i}, q_{i}\right), i=1,2,3$. That is, in the time evolution of the particle trajectories, the variables cover some range of values, and we must estinale that range. We recall that we have scaled the motion so that $V \sim 1$, in particular $V_{\theta} \sim 1$. We readily find from (8), (13), (16) and (17) that

$$
v_{\theta}=-q_{3} /(\varepsilon r) \text {. }
$$

Since we assume that we are far from the axis $r=0, \varepsilon r \sim 1$ and $1 /(\varepsilon r)$ $\sim 1$, so that we conclude $q_{3}=O(1)$. The analogous argument applied to $v_{r}$ and $V_{z}$ leads to the conclusion that $p_{2}$ and $p_{3}$ also are $O(1)$ in $\varepsilon$. Now both $S$ and $\psi$ are coordinates of points in physical space and we expect them both to have the range $O(1)$ in $\varepsilon$. Thus, we see from (16) that $\varepsilon^{2} P_{1}$ and $\varepsilon q_{2}$ are $O(1)$. The ranqe of $\theta$ and $q_{1}$ are essentially the 
same and may be large in $\varepsilon$ if the particle makes a large number of transits around the torus. Since the Hamiltonian is periodic in $\theta$, and hence $q_{1}$, large values of either variable leave the Hamiltonian and all its derivatives $O(1)$. We treat $q_{1}=O(1)$ although it may become large. In summary, the combinations $\varepsilon^{2} p_{1}, q_{1}, p_{2}, \varepsilon q_{2}, p_{3}$ and $q_{3}$ are all $o(1)$. These properties are essential to the arguments that follow.

Th. will hernme slear shortly that $\left(p_{3}, q_{3}\right)$ arc essentially lie component of $\mathrm{v}_{1}$, the gyro rotation velocity, $\mathrm{p}_{2}$ is related to $\mathrm{v}_{\|}$while $\varepsilon q_{2}=S$ is the position of a particle on a magnetic field line, and $\left(p_{1}, q_{1}\right)$ are the canonical angular momentum and angular position, rspectively. We may also interpret $\varepsilon^{2} p_{1}$ as essentially the flux coordinate of the particle. We may insert (16) and (17) ito (14) to find the new Hamiltonian as

$$
\begin{aligned}
H= & (1 / 2)\left\{E(\psi, S)\left(p_{3}-\varepsilon q_{3}^{2} \lambda, \psi \psi / 2-A_{\psi}(\psi, s, \theta)\right)^{2}\right. \\
& \left.+G(\psi, S)\left(p_{2}+q_{3} \lambda, S-\varepsilon q_{3}^{2} \lambda, \psi S / 2-A_{S}(\psi, S, \theta)\right)^{2}+R(\psi, S) q_{3}^{2}\right\}
\end{aligned}
$$

where.

$$
\begin{aligned}
& E=\psi_{, \varepsilon r}^{2}+\psi_{, \varepsilon z}^{2} \\
& G=s_{, \varepsilon \cdot r}^{2}+s_{, \varepsilon z}^{2} \\
& R=1 /(\epsilon L)^{2}
\end{aligned}
$$

and

$$
\hat{\dot{r}} A_{r}+\hat{z} A_{z}=A_{\psi}(\nabla \psi / \varepsilon)+A_{S}(\bar{\nabla} S / \varepsilon)
$$

E, G, R are first evaluated as functions of $\varepsilon r, \varepsilon z$ and then $\varepsilon r, \varepsilon z$ are considered as functions $\psi$ and $S$. Since $\nabla \psi / \varepsilon$ and $\nabla S / \varepsilon$ are both $O(1), A_{\psi}$ and $A_{S}$ are $O(1)$ and they are treated similarly first as functions of 
$\varepsilon r, \varepsilon z, \theta$ and then as functions of $\psi, S$, and $\theta$. Finally in (18) we employ (16) to replace $\psi, S$, and $\theta$ in $H$ by the new canonical variables $\left(p_{i}, q_{i}\right), i=1,2,3$. If $A_{\psi}=A_{S}=0$ so that no nonaxisymetric fields appear, the Hamiltonian is independent of $q_{1}$ so that $p_{1} \equiv$ const. and axisymnetry is preserved. In the usual treatment the Clebsch variables $\alpha, \beta, S$ are used as coordinates, $B=\nabla \alpha \times \unrhd \beta$ and the coordinates $\theta$ enters in a nonignorable manner in $\alpha(r, \theta, z)$ or $\beta(r, \theta, z)$.

We see that the essential structure of our Hamiltonian is

$$
\mathrm{H}=\mathrm{H}\left(\varepsilon^{2} \mathrm{p}_{1}, \mathrm{q}_{1}, \mathrm{p}_{2}, \varepsilon \mathrm{q}_{2}, \mathrm{p}_{3}, \mathrm{q}_{3}, \varepsilon\right)
$$

where derivatives of $\mathrm{H}$ with respect to its arguments are all $O(1)$. We may rewrite Hamilton's equations as

$$
\begin{array}{ll}
\left(\varepsilon \dot{p}_{1}\right)=-\varepsilon^{2}\left(\frac{\partial H}{\partial q_{1}}\right) & \dot{q}_{1}=\varepsilon^{2}\left(\frac{\partial H}{\partial \varepsilon^{2} p_{1}}\right) \\
\dot{p}_{2}=-\varepsilon\left(\frac{\partial H}{\partial \varepsilon q_{2}}\right) & \left(\varepsilon \dot{q}_{2}\right)=\varepsilon \frac{\partial H}{\partial p_{2}} \\
\dot{p}_{3}=-\frac{\partial H}{\partial \dot{q}_{3}} & \dot{q}_{3}=\frac{\partial H}{\partial p_{3}} .
\end{array}
$$

Hence, over a time interval $o(1) \Delta p_{3}$ and $\Delta q_{3} \sim 1$ while $\Delta p_{2}$ and $\Delta\left(\varepsilon q_{2}\right) \sim$ $\varepsilon$, but $\Delta \varepsilon^{2} p_{1}$ and $\Delta q_{1} \sim r^{2}$. Provided $p_{3}$ and $q_{3}$ remain $O(1)$ over the longer time interval $O(l / \varepsilon), \Delta p_{2}$ and $\Delta\left(\varepsilon q_{2}\right) \sim 1$. Finally over the longest time interval $O\left(1 / \varepsilon^{2}\right), \Delta \varepsilon^{2} p_{1}$ and $\Delta q_{1}$ are $O(1)$. Thus, our variables $\left(p_{i}, q_{i}\right)$ have decomposed the motion into motion on the three distinct time scales $1,1 / \varepsilon$, and $1 / \varepsilon^{2}$. This decomposition holds even in the axisymmetric case $A_{S}=A_{\psi}=0$.

We are now prepared to construct the various adiabatic invariants 
following Gardner's procedure. If we hold $\varepsilon^{2} p_{1}, q_{1}, p_{2}$ and $\varepsilon q_{2}$ fixed we see that the curves $H=$ const. are ellipses in the $p_{3}, q_{3}$ plane. We may distort the ellipses into circles by an affine transformation in the $p_{3}, q_{3}$ plane and we may generate such an affine transformation by a canonical transformation in the $p_{3}, q_{3}$ plane. We may also, then, extend the transformation into the full $\left(p_{i}, q_{i}\right), i=1,2,3$ space. Specifically, we introduce the generator of the transformation from $\left(p_{i}, q_{i}\right), i=1,2,3$ to $\left(\bar{p}_{i}, \bar{q}_{i}\right), i=1,2,3$,

$$
F=\left(\alpha \bar{p}_{3}+A_{\psi}\right)\left(q_{3}+\gamma\right)+\bar{p}_{1} q_{1}+\bar{p}_{2} q_{2}
$$

where

$$
\begin{aligned}
E \alpha^{2} & =\left(B_{T}^{2}+B_{p}^{2}\right)^{1 / 2} \\
r & =G\left(\bar{p}_{2}=A_{S}\right) \lambda_{, S} /\left(G \lambda_{, S}^{2}+R\right)
\end{aligned}
$$

and the several functions $\alpha, \gamma, E, G, R, B_{p^{\prime}} B_{T}, \lambda, S, A_{\psi}$ and $A_{S}$ are first evaluated as spatial functions of $\psi, s, \theta$ followed by the replacements

$$
\begin{aligned}
& \psi=\varepsilon{ }^{2} \bar{p}_{1}+\varepsilon q_{3} \\
& s=\varepsilon q_{2} \\
& \theta=q_{1}+\lambda(\psi, s)+\varepsilon \bar{p}_{3}-\varepsilon q_{3^{\lambda}, \psi^{\prime}}
\end{aligned}
$$

We find easily

$$
\begin{aligned}
\varepsilon^{2} p_{1} & =\varepsilon^{2} \bar{p}_{1}+o\left(\varepsilon^{2}\right) \\
q_{1} & =\bar{q}_{1}+o\left(\varepsilon^{2}\right) \\
p_{2} & =\bar{p}_{2}+o(\varepsilon)
\end{aligned}
$$




$$
\begin{aligned}
\varepsilon q_{2} & =\varepsilon \bar{q}_{2}+O(\varepsilon) \\
p_{3} & =\alpha \vec{p}_{3}+A_{\psi}+O(\varepsilon) \\
q_{3} & =\bar{q}_{3} / \alpha-\gamma+O(\varepsilon)
\end{aligned}
$$

so that the barred and original variables are of the same order in $\varepsilon$. We could easily but tediously evaluate the error terms to any desired order in $\varepsilon$. The transformed Hamiltonian becomes

$$
H=(1 / 2)\left(\bar{p}_{3}^{2}+\bar{q}_{3}^{2}\right) B+(1 / 2) G\left(\bar{p}_{2}-A_{S}\right)^{2} B_{P}^{2} / B^{2}+O(\varepsilon)
$$

where

$$
B=\left(B_{p}^{2}+B_{T}^{2}\right)^{1 / 2}
$$

and $B_{p}, B_{T}, B, G$ and $A_{S}$ are first evaluated as functions of $\psi, \lambda, \theta$ followed by the replacements $\psi=\varepsilon^{2} \bar{p}_{1}, s=\varepsilon \bar{q}_{2}, \theta=\bar{q}_{1}+\lambda(\psi, S)$.

We may now readily repeat the process. The terms proportional to $\varepsilon$ in the Hamiltonian (23) are a third degree polynomial in $\left(\bar{p}_{3}, \bar{q}_{3}\right)$. With an appropriate canonical transformation (see the appendix where an analogous transformation is carried out in some detail) we find

$$
\begin{aligned}
& H=(1 / 2)(B+\varepsilon L)\left(\bar{p}^{2}{ }_{3}+\overline{\bar{q}}^{2}{ }_{3}\right)+(1 / 2) G\left(\overline{\bar{p}}_{2}-\mathrm{A}_{S}\right)^{2} \mathrm{~B}_{\mathrm{p}}^{2} / \mathrm{B}^{2} \\
& +\varepsilon M\left(\overline{\bar{p}}_{2}, \varepsilon \overline{\bar{q}}_{2}, \varepsilon \overline{\bar{p}}_{1}, \overline{\bar{q}}_{1}\right)+O\left(\varepsilon^{2}\right)
\end{aligned}
$$

where $B_{p}, B_{T}, B, L, G$, and $A_{S}$ are evaluated at $\psi, S, \theta$ followed by the replacements $\psi=\varepsilon \varepsilon^{2} \overline{\overline{\mathrm{p}}}_{1}, \mathrm{~S}=\varepsilon \overline{\overline{\mathrm{q}}}_{2}, \theta=\overline{\overline{\mathrm{q}}}_{1}+\lambda(\psi, S)$. After $N$ steps we conclude

$$
H=H_{N}\left(\varepsilon, \tilde{p}_{1}, \tilde{q}_{1}, \tilde{p}_{2}, \varepsilon \tilde{q}_{2}, \tilde{p}_{3}^{2}+\tilde{q}_{3}^{2}, \varepsilon\right)+O\left(\varepsilon^{N}\right) .
$$


We infer from Hamilton's equations that

$$
(d / d t)\left(\tilde{p}_{3}+\tilde{q}_{3}^{2}\right)=O\left(\varepsilon^{N}\right)
$$

so that $\tilde{p}_{3}^{2}+\tilde{q}_{3}^{2}$ is an adiabatic invariant which changes only by a small amount over very long time intervals. For very long time intervals we may replace $\tilde{p}_{3}^{2}+\tilde{q}_{3}^{2}$ by the constant $2 \mu$, which, to lowest order, is twice the usual magnetic moment. We shall employ only the lowest order form (23) although we need to know that in principle a result such as (25) is possible, where $H_{N}$ is, to lowest order in $\varepsilon$, the form given in (23). Clearly over the time interval $O\left(\varepsilon^{-N}\right), \Delta\left(\tilde{p}^{2} 3+\right.$ $\left.\tilde{q}_{3}^{2}\right)=O\left(\varepsilon^{N-M}\right)$, and we might pick $N$ and $M$ as we need for a given situation.

Since we may consider $\tilde{p}_{3}^{2}+\tilde{q}_{3}^{2}$ as a constant over some long time interval we have reduced the Hamiltonian in (25) to depend on the two pair of canonically conjugate variables $\left(\tilde{p}_{1}, \tilde{q}_{1}\right)$ and $\left(\tilde{p}_{2}, \tilde{q}_{2}\right)$. Further the Hamiltonian depends on these variables in the form

$$
\mathrm{H}=\mathrm{H}\left(\varepsilon \tilde{\mathrm{p}}_{1}, \tilde{q}_{1}, \tilde{\mathrm{p}}_{2}, \varepsilon \tilde{q}_{2}, \varepsilon\right)
$$

so that again there is a separation of time scales with $\left(\varepsilon \tilde{p}_{1}, \tilde{q}_{1}\right)$ vaying on the $O\left(1 / \varepsilon^{2}\right)$ time scale while $\left(\tilde{p}_{2}, \varepsilon \tilde{q}_{2}\right)$ vary on the $O(1 / \varepsilon)$ scale. Thus, we have again the masihility of the mostrustion of a second adiabatic invariant. In order to construct the invariant we need not only the time scale separation, but also periodic orbits in the fast variables. For example, we started the construction of the magnetic moment with the observation that the orbits in the $\left(p_{3}, q_{3}\right)$ plane were ellipses, a property which automatically gives periodic orbits in these variables with all the other variables held fixed. We 
must now investigate the implications of the periodicity condition in greater detail for the two distinct cases.

We may introduce the more or less standard guiding center Hamiltonian

$$
\begin{aligned}
H_{0}=\mu B\left(\varepsilon^{2} p_{1}, \varepsilon q_{2}\right)+(1 / 2) G\left(\varepsilon^{2} p_{1}, \varepsilon q_{2}\right) \times\left(p_{2}-A_{S}\left(\varepsilon{ }^{2} p_{1}, \varepsilon q_{2}, q_{1}+\lambda\left(\varepsilon{ }^{2} p_{1}, \varepsilon q_{2}\right)\right)\right)^{2} \\
\times B_{p}^{2}\left(\varepsilon^{2} p_{1}, \varepsilon q_{2}\right) / B^{2}\left(\varepsilon{ }^{2} p_{1}, \varepsilon q_{2}\right)
\end{aligned}
$$

so that the full Hamiltonian is

$$
H=H_{0}+\varepsilon H_{1}\left(\varepsilon{ }^{2} p_{1}, \dot{q}_{1}, p_{2}, \varepsilon q_{2}, \varepsilon\right) ;
$$

and we shall see that $\mathrm{H}_{1}$ does not materially alter our conclusions. If we hold $\varepsilon^{2} p_{1}$ and $q_{1}$ fixed we see that the motion in the $p_{2}, \varepsilon q_{2}$ plane allows two distinct classes of orbits. In the first class $\varepsilon q_{2}$, which to leading order is $\mathrm{S}$, is bounded above and below. These orbits represent particles lrapped by maxima or minima in $B$ and the orhita ' represent the trapped particles which do not encircle the magnetic axis. The orbits are often called banana orbits as their shape approximates a banana in the $\dot{p}_{2}, \varepsilon q_{2}$ plane. Since $\varepsilon q_{2}$ is bounded, $p_{2}$ is also bounded and the orbits are automatically periodic in the $\left(p_{2}, \varepsilon q_{2}\right)$ planes. For the second class of orbits $\varepsilon q_{2}$ increases or decreases monotonically in time and the orbit is untrapped and the particles are often called passing particles. These particles encircle the magnetic axis. We examine the trapped particles first.

When we introduced the magnetic moment adiabatic invariant we 
could carry out the process explicitly since the Hamiltonian to any order in $\varepsilon$ was a polynomial in $p_{3}$ and $q_{3}$. There is, however, an implicit process to carry out the adiabatic invariant construction as given by Gardner. For our Hamiltonian (26) and (27) let us first hold $\varepsilon^{2} p_{1}$ and $q_{1}$ fixed and onsider the $p_{2}, \varepsilon q_{2}$ plane alone. We may construct canonical tansformations in the plane starting from the characterization of a canonical transformation as an area preserving transformation. We introduce the area inside the curve $\mathrm{H}=$ const., or equivalently the action,

$$
J=\underset{H=\text { const. }}{J} \mathrm{P}_{2} \mathrm{~d} \varepsilon \mathrm{q}_{2}
$$

and the associated angle variable $\theta$ such that area is preserved

$$
\mathrm{dp}_{2} \cdot \mathrm{d} \varepsilon \mathrm{q}_{2}=\mathrm{dJ} d \theta \text {. }
$$

Such a transformation is canonical and there is a qenerator $G$ such that

$$
p_{2} d \varepsilon q_{2}=-\theta d J+d G\left(\varepsilon q_{2}, J\right) \text {. }
$$

Now $G$ in fact is a function of $\varepsilon^{2} p_{1}, q_{1}$ also so that

$$
G=G\left(\varepsilon q_{2}, J, \varepsilon^{2} p_{1}, q_{1}\right)
$$

and we may then construct a canonical transformation in the full space by the use of the generator

$$
F=G\left(\varepsilon q_{2}, J, \varepsilon \bar{p}_{1}, q_{1}\right) / \varepsilon+\bar{p}_{1} q_{1}
$$

... For such a transformation

$$
\varepsilon^{2} p_{1}=\varepsilon^{2} \bar{p}_{1}+O(\varepsilon)
$$




$$
q_{1}=\bar{q}_{1}+o(\varepsilon)
$$

and to leading order $\left(p_{1}, q_{1}\right)$ and $\left(\bar{p}_{1}, \bar{q}_{1}\right)$ are interchangeable. Further, by construction

$$
H=H\left(J, \varepsilon \bar{p}_{1}, \bar{q}_{1}\right)+O(\varepsilon) \text {. }
$$

If we now iterate the transformation in the usual manner we find that after $\mathrm{N}$ steps

$$
H=H\left(J^{(N)}, \varepsilon^{2} p I^{N}, q f^{N}\right),+O\left(\varepsilon^{N}\right),
$$

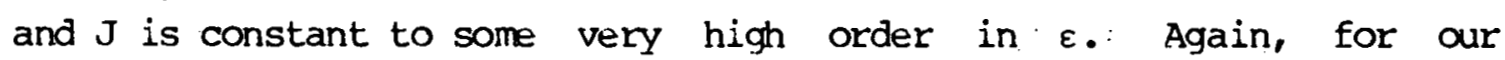
purposes it suffices to know that (30) is possible, we use only (29). For the trapped particles we see from (26)

$$
\left.P_{2}=A_{S} \pm(2(H-\mu B+O(\varepsilon)) / G)\right)^{1 / 2} B / B_{p}
$$

where the + occurs for $\varepsilon q_{2}$ increasing and the - sign for $\varepsilon q_{2}$ decreasing. The adiabatic invariant to lowest order in $\varepsilon$ is then

$$
\begin{aligned}
& J=\int_{S_{\min }}^{S_{\max }}\left(A_{S^{+}}((2 H-2 \mu B) / G)^{1 / 2} B / B_{p}\right) d z q_{2} \\
& +\int_{S_{\max }}^{S_{\min }}\left(A_{S}-((2 H-2 \mu \mathrm{B}) G)^{1 / 2} \mathrm{~B} / \mathrm{B}_{\mathrm{p}}\right) d \varepsilon \mathrm{q}_{2} \\
& J=2 \int_{S_{\min }}^{S_{\max }}(2(H-\mu B) / G)^{I / 2} B / B_{p} d E \varphi_{2}
\end{aligned}
$$


In (31). $\mathrm{B}_{\mathrm{p},} \mathrm{B}$, and $\mathrm{G}$ are functions of $\varepsilon{ }^{2} \mathrm{p}_{1}$ and $\varepsilon q_{2}$ only; there is no $\mathrm{q}_{1}$ dependence. If invert (31) to obtain $\mathrm{H}$ we find that the Hamiltonian corresponding to $(30)$ is

$$
\mathrm{H}=\mathrm{H}_{0}\left(\mu, \mathrm{J}, \varepsilon^{2} \mathrm{P}_{1}\right)+\mathrm{O}(\varepsilon),
$$

so that if we now apply the property that $\mathrm{H}=$ const. on the trajectory we see that $e^{2} p_{1}$ conot. $+O(c)$ for an arbitracily long time. Sinno $\varepsilon^{2} \mathrm{p}_{1}$ is the flux coordinate of the particle we conclude the particle flux cordinate is bounded within a small interval for a very long time. Hence any trapped particle drifts are essentially nonadiabatic in their very nature. At the end of this section we comment on this point further.

As a preliminary to the study of the passing particles, we must examine the vector potential further. We may normalize the function $S(r, z)$, see (7), so that all flux surfaces $\psi=$ constant corresponds to the range of values $0 \leq S \leq 1$. Henoe, $\mathrm{B}$ considered as a function of $\psi$, $S$, and $\theta$ is periodic of period 1 in $S$ and periodic of period $2 \pi$ in $\theta$. As follows from $(4), \lambda(r, z)$ is not single valued but if we consider $\lambda=$ $\lambda(\psi, S)$ then

$$
\lambda(\psi, S+i)=\lambda(\psi, \bar{S})+\Lambda(\psi)
$$

If we consider $A_{S}$ as a function of $\psi, S$, and $\theta$ and introduce the variables $\left.\psi=\varepsilon^{2} \mathrm{p}_{1}, \mathrm{~s}=\varepsilon \mathrm{q}_{2}, \theta=q_{1}+\lambda\left(\varepsilon^{2} \mathrm{p}_{1}, \varepsilon \mathrm{q}_{2}\right)\right)$, then

$$
\begin{aligned}
& A_{S}\left(\varepsilon^{2} p_{1}, \varepsilon q_{2}+n, q_{1}+\lambda\left(\varepsilon^{2} p_{1}, \varepsilon q_{2}+n\right)\right) \\
& \quad=A_{S}\left(\varepsilon^{2} p_{1}, \varepsilon q_{2}, q_{1}+\lambda\left(\varepsilon^{2} p_{1}, \varepsilon q_{2}\right)+n \Lambda\left(\varepsilon{ }^{2} p_{1}\right)\right)
\end{aligned}
$$


Thus, the vector potential is not periodic in $\varepsilon q_{2}$ of period 1 unless $\mathrm{n} \Lambda\left(\varepsilon^{2} \mathrm{p}_{1}\right)$ is an integral multiple of $2 \pi$. We see that we must consider the effects of resonances of the motion the short way around the torus and the motion the long way around the torus.

Let us consider first the simplest case in which $\Lambda(\psi)$ is independent of $\psi$ and there is a positive integer $\mathrm{N}$ such that $\mathrm{N} \Lambda$ is an integral multiple of $2 \pi$; further let $N$ be the smallest such positive integer. It then follows that in the $\left(\mathrm{p}_{2}, \varepsilon q_{2}\right)$ plane the orbit is periodic if $\varepsilon q_{2}$ covers the range $0<-\varepsilon q_{2} \leq N$. We may then apply analysis analogous to the trapped particle case and if we take, without Ioss of generality, the + sign in (31) we find the lowest order adiabatic invariant

$$
J=\int^{N} d \varepsilon q_{2}\left(A_{S}\left(\varepsilon^{2} p_{1}, \varepsilon q_{2}, q_{1}+\lambda\left(\varepsilon^{2} p_{1}, \varepsilon q_{2}\right)\right)+(2(H-\mu B) / G){ }^{1 / 2} B / B_{p}\right\}
$$

which we may invert to find the drift hamiltonian

$$
\dot{H}=H\left(\mu, J, \varepsilon^{2} p_{1}, q_{1}\right)+O(\varepsilon) \text {. }
$$

We see that the drift Hamiltonian contains $q_{1}$ nontrivially so that the trajectory $\mathrm{H}=$ const. admits the possibility of large long term drifts. Thus, the nonaxisymmetric fields introduce the possibility of large, nontrivial drifts. We may readily show, however, that the drifts need not be large. Suppose $\mathrm{N} \Lambda=2 \pi \mathrm{M}$, where $\mathrm{M}$ and $\mathrm{N}$ are relatively prime and $N$ is large, but $N \varepsilon \ll 1$. In this case

$$
\int_{0}^{N} d \varepsilon q_{2} A_{S}\left(\varepsilon^{2} p_{1}, \varepsilon q_{2}, q_{1}+\lambda\left(\varepsilon^{2} p_{1}, \varepsilon q_{2}\right)\right)
$$




$$
\begin{aligned}
& =\sum_{r=0}^{N-1} \int_{0}^{1} d \varepsilon q_{2} A_{S}\left(\varepsilon^{2} p_{1}, \varepsilon q_{2}, q_{1}+\lambda\left(\varepsilon^{2} p_{1}, \varepsilon q_{2}\right)+r \Lambda\right) \\
& =\frac{N}{2 \pi} \int_{0}^{1} d \varepsilon q_{2} \int_{0}^{2 \pi} d q_{1} A_{S}\left(\varepsilon^{2} p_{1}, \varepsilon q_{2}, q_{1}+\lambda\left(\varepsilon^{2} p_{1}, \varepsilon q_{2}\right)\right)+o(1),
\end{aligned}
$$

or with (6.)

$$
\int_{0}^{N} d \varepsilon q_{2} A_{S}\left(\varepsilon^{2} p_{1}, \varepsilon q_{2}, q_{1}+\lambda\left(\varepsilon^{2} p_{1}, \varepsilon q_{2}\right)\right)=o(1),
$$

where the $O(1)$ estimate in (38) estimates the error as $\mathrm{N}$ becomes large. The adiabatic invariant (36.) would then become

$$
\mathrm{J}=\mathrm{N} \prod_{0}^{1} \mathrm{~d} \varepsilon \mathrm{q}_{2}(2(\mathrm{H}-\mathrm{HB}) / \mathrm{G})^{1 / 2} \mathrm{~B} / \mathrm{B}_{\mathrm{P}}+\mathrm{O}(1)
$$

so that for $\mathrm{N}$ large and $\varepsilon$ smail the drifts would again become bounded and small, since $q_{1}$ is missing in the leading order term in (39). We may relax slightly the constraint that $\Lambda$ be constant and $\Lambda / 2 \pi$ a rational number. 'We make the slightly weaker assumption that $\Lambda / 2 \pi$ be close to a rational; specifically $-\varepsilon<\Lambda / 2 \pi-M / N<\varepsilon$. In this case we can readily add an $O(\varepsilon)$ correction to $H_{0}$ and subtract it from $\mathrm{H}_{1}$ in (20), (27) so that we can take $\Lambda \equiv 2 \pi \mathrm{M} / \mathrm{N}$. Since none of our results depends on $\mathrm{H}_{1}$, we see that we may apply the results just given if $\Lambda(\psi)$ remains close (in $\varepsilon$ ) to a given rational number.

When $\Lambda^{\prime}(\psi)$ is not small the adiabatic invariant sonstruction fails. Although there may be approximately periodic orbits, there is not the nested family of periodic orbits necessary to make the construction work. One can see from the results just obtained that one would expect rapid particle drift away from surfaces where $\Lambda(\psi) / 2 \pi$ is 
approximated by a rational number with small denominator while the drifts would be very slow, if any at all, near surfaces where the rational approximation had a large denominator. Clearly such a process does not lend. itself to a continuous, let alone differentiable, description. It is apparent that without the adiabatic invariant we have the real possibility of a large drift formed from very long time cumulation of small drifts.

We note that a standard canonical transformation makes the whole process relatively easy to compute. Since the Hamiltonian $H$ is time independent, $H=$ const., and we can eliminate two of the variables. (6) For the general system with two pair of canonically conjugate variables,

$$
p_{1} d q_{1}+p_{2} d q_{2}-H d t=-d(H t)+t d H+p_{1} d q_{1}-\left(-p_{2}\right) d q_{2} \text {, }
$$

so that if we invert $H=$ const. to yield $-p_{2}=-p_{2}\left(p_{1}, q_{1}, H, q_{2}\right)$ we may consider $-p_{2}$ as the "Hamiltonian" of the motion, $q_{2}$ as the "time" and $\left(p_{1}, q_{1}\right)$ and $(t, H)$ as the two pair of canonically conjugate variables. We thus find

$$
\begin{array}{ll}
\frac{d \mathrm{H}}{d q_{2}}=0, & \frac{d t}{d q_{2}}=\frac{\partial q_{2}}{\partial H} \\
\frac{d p_{1}}{d q_{2}}=\frac{\partial p_{2}}{\partial q_{1}}, & \frac{\partial q_{1}}{d q_{2}}=-\frac{\partial p_{2}}{\partial p_{1}}
\end{array}
$$

and in our case from (31) and with the reintroduction of physical variables $\psi, S, \theta$ 


$$
\begin{aligned}
\frac{d \psi}{d S} & =\varepsilon A_{S, \theta}(\psi, S, \theta+\lambda(\psi, S)) \\
\frac{d \theta}{d S} & =-\varepsilon\left\{A_{S, \psi}(\psi, S, \theta+\lambda(\psi, S))+\lambda, \psi^{A} S, \theta(\psi, S, \theta+\lambda(\psi, S))\right. \\
& \left.+\frac{\partial}{\partial \psi}\left((2(H-\mu B(\psi, S)) / G(\psi, S))^{1 / 2} B(\psi, S) / B_{p}(\psi, S)\right)\right\}
\end{aligned}
$$

The system (40) is reminiscent of a Mathieu or Hill's equation for which resonances and unbounded orbits may or may not occur. The unbounded orbits may appear even in cases where adiabatic invariants exist to all orders, just as for the Mathieu equation. Finally it shold be noted that if one must follow the orbits for very long times, $\varepsilon \Delta S \gg 1$, then a higher order guiding center Hamiltonian is needed to give meaningful results. We could apply the system $(40)$ to trapped particles as well although at the turning points of the trapped particles $(40)$ becomes singular. It is by no means obviously correct that nonadiabatic effects arising from (40) are reliable when (40) itself is based on the magnetic monent adiabatic invariant. 
IV. The Guiding. Center Expansion for $\mathrm{B}_{\mathrm{P}}$ Small

If we examine the Hamiltonian obtained for the guiding center motion in the general case (26), we see that as $B_{p}$ becomes small the Hamiltonian becomes badily degenerate. We reexamine the entire problem for $\mathrm{B}_{\mathrm{P}} \sim \varepsilon$. The coordinates introduced in the previous section (16), (17) are not appropriate to describe the motion in this case. We introduce a new set of coordinates appropriate to the present scaling and consistent with axial symmetry, if present. We are aqain able to decompose the motion into motions on three distinct time scales, the fastest of which again is the gyro rotation about the guiding center. In contrast with the previous section in which $p_{\theta}$ varied on the slowest time scale, now $p_{\theta}$ varies. on the intermediate time scale. Before, the flux coordinate of a particle was essentially $p_{\theta}$ and was canonically conjugate to the toroidal angle coordinate. Now, the flux is conjugate to another coordinate characterizing a given field line. The drift surfaces are now large orbits with or without axial symetry. A second adiabatic invariant always exists, but the non-axisymmetric magnetic fields have only slight effects ur' the drift orhits by reconnecting orbits near critical points in the appropriate phase plane.

We recall that if we modify the $\theta$ component of (8) to become

$$
A_{\theta}^{\prime}=\psi(\varepsilon r, \varepsilon z) /(\varepsilon r)
$$

then $\mathrm{B}_{\mathrm{T}}$ as given by (9) is unaffected but

$$
B_{p}=\varepsilon(-\hat{r} \psi, \varepsilon z /(\varepsilon r)+\hat{z} \psi, \varepsilon, r /(\varepsilon r))
$$

and

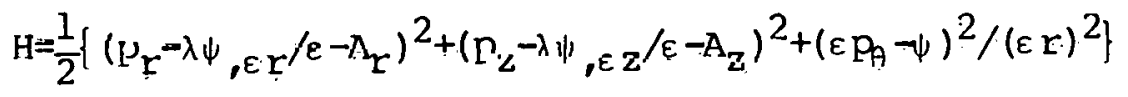


We introduce a generator of a canonical transformation essentially equivalent to the introduction of $(\alpha, \beta, s)$ coordinates of the toroidal field alone

$$
F=\theta p_{1}+(\psi / \varepsilon) p_{2}+(\lambda / \varepsilon) p_{3}-p_{2} p_{3}
$$

from which it follows that

$$
\begin{aligned}
& \theta=q_{1} \\
& \psi=\varepsilon q_{2}+\varepsilon p_{3} \\
& \lambda=\varepsilon p_{2}+\varepsilon q_{3}
\end{aligned}
$$

and

$$
\begin{aligned}
& p_{\theta}=p_{1} \\
& \mathrm{P}_{\mathrm{r}}-\lambda{ }_{{ }, \varepsilon \mathrm{r}^{/ \varepsilon}=-\mathrm{q}_{3}{ }, \varepsilon, \mathrm{r}}+\mathrm{P}_{3}{ }^{\lambda}, \varepsilon \mathrm{r} \\
& \mathrm{p}_{\mathrm{z}}-\lambda{ }_{,}, \varepsilon \mathrm{z} / \varepsilon=-\mathrm{q}_{3} \psi, \varepsilon \mathrm{z}+\mathrm{p}_{3^{\lambda}, \varepsilon \mathbf{z}}
\end{aligned}
$$

Just as in the previous section the mnition that $\underset{\sim}{ }$ be of ouder 1 in $\varepsilon$ leads to the conclusion that $q_{3}$ and $p_{3}$ are both $O(1)$. Since we wish $\psi$ and $\lambda$ to span a range of values $O(1)$ we infer that $\varepsilon q_{2}$ and $\varepsilon p_{2}$ must be $O(1)$, and since $V_{\theta}$ must be $O(1)$ we conclude $\varepsilon p_{1}$ be $O(1)$. The Hamiltonian (14a) becomes

$$
\begin{aligned}
H=(1 / 2) & \left\{E(\psi, \lambda)\left(q_{3}+A_{\psi}(\psi, \lambda, \theta)\right)^{2}\right. \\
+ & 2 F(\lambda, \theta)\left(q_{3}+A_{\psi}(\psi, \lambda, \theta)\right)\left(p_{3}-A_{\lambda}(\psi, \lambda, \theta)\right) \\
+ & \left.G(\lambda, \theta)\left(p_{3}-A_{\lambda}(\psi, \lambda, \theta)\right)^{2}+R(\lambda, \theta)\left(\varepsilon p_{1}-\varepsilon q_{2}-\varepsilon p_{3}\right)^{2}\right\}
\end{aligned}
$$

where $\psi, \lambda, \theta$ in (44) are replaced by (42) and 


$$
\begin{aligned}
& E(\psi, \lambda)=(\psi, \varepsilon r)^{2}+(\psi, \varepsilon z)^{2} \\
& F(\psi, \lambda)=-\left(\psi, \varepsilon r^{\lambda}, \varepsilon r+\psi, \varepsilon z^{\lambda}, \varepsilon z\right) \\
& G(\psi, \lambda)=(\lambda, \varepsilon r)^{2}+(\lambda, \varepsilon z)^{2} \\
& R(\psi, \lambda)=1 /(\varepsilon r)^{2} \\
& \hat{r} A_{r}+\hat{z} A_{z}=A_{\psi}(\psi, \lambda, \theta) . \nabla \psi / \varepsilon+A_{\lambda}(\psi, \lambda, \theta) \nabla \lambda / \varepsilon
\end{aligned}
$$

and the underlying structure is

$$
H=H\left(\varepsilon p_{1}, q_{1}, \varepsilon p_{2}, \varepsilon q_{2}, p_{2}, q_{2}, \varepsilon\right) \text {. }
$$

For a Hamiltonian of this structure, (46),

$$
\begin{array}{ll}
\dot{\varepsilon} \mathrm{p}_{1}=-\varepsilon \partial \mathrm{H} / \partial \mathrm{q}_{1}, & \dot{q}_{1}=\varepsilon \partial \mathrm{H} / \partial\left(\varepsilon \mathrm{p}_{1}\right), \\
\dot{e \dot{p}_{2}}=-\varepsilon{ }^{2} \partial \mathrm{H} / \partial\left(\varepsilon \mathrm{q}_{2}\right), & \varepsilon \dot{\mathrm{q}}_{2}=\varepsilon^{2} \partial \mathrm{H} / \partial \varepsilon \mathrm{p}_{2}, \\
\dot{\mathrm{p}}_{3}=-\partial \mathrm{H} / \partial \mathrm{q}_{3}, & \dot{\mathrm{q}}_{3}=\partial \mathrm{H} / \partial \mathrm{p}_{3},
\end{array}
$$

so that $\left(p_{3}, q_{3}\right)$ vary on the fastest $O(1)$ time scale, $\left(\varepsilon p_{1}, q_{1}\right) \equiv\left(\varepsilon p_{\theta}, \theta^{\prime}\right)$. varies on the next sinwer $O(1 / \varepsilon)$ time scale, while $\left(\varepsilon p_{2}, \varepsilon q_{2}\right)$ vary on the slowest $O\left(1 / \varepsilon^{2}\right)$ time scale. We see that the variable structure is entirely different from the previous section. It is still true that $\left(p_{3}, q_{3}\right)$ are essentially the components of the gyro velocity, but the other variables are completely rearranged. In particular, $p_{1}$ is st1ll $\mathrm{p}_{\theta}$, but it is no longer identified with magnetic flux, rather $\mathrm{p}_{\theta}$ is essentially $v_{\|}$. The canonically conjugate coordinate $q_{1}$ is essentially $\theta$. The variable $\lambda$ did not occur before (indeed in the previous section $\lambda \equiv 0$ corresponding to a mirror machine or pure 2 pinch is admissible). It is still true that $\psi$ varies on the slowest time scale, but its 
canonically conjugate oordinate switches from the toroidal angle $\theta$ to the field line cordinate $\lambda$.

We may now start the construction of the magnetic moment adiabatic invariant and guiding center motion Hamiltonian. We introduce the generator

$$
F=\left(\alpha \bar{p}_{3}+A_{\lambda}\right)\left(q_{3}+A_{1}\right)+B \bar{p}_{3}^{2} / 2+\bar{p}_{1} q_{1}+\bar{p}_{3} q_{2}
$$

where

$$
\begin{aligned}
& \alpha^{2}=E / B \\
& B=F / B \\
& B=B_{T}=\left(E G-F^{2}\right)^{1 / 2}
\end{aligned}
$$

and all functions of space in (47), (48) are evaluated at $\psi, \lambda, \theta$ followed by the replacements $\psi=\varepsilon \bar{p}_{2}, \lambda=\varepsilon q_{2}$ and $\theta=q_{1}$. We readily find that

$$
\begin{aligned}
& \varepsilon \mathrm{p}_{1}=\varepsilon \overline{\mathrm{p}}_{1}+O(\varepsilon) \\
& \mathrm{q}_{1}=\overline{\mathrm{q}}_{1} \\
& \varepsilon \mathrm{p}_{2}=\varepsilon \overline{\mathrm{p}}_{2}+O(\varepsilon) \\
& \varepsilon \mathrm{q}_{2}=\varepsilon \overline{\mathrm{q}}_{2}+O(\varepsilon) \\
& \mathrm{p}_{3}=\alpha \overline{\mathrm{p}}_{3}+\Lambda_{\lambda}+O(\varepsilon) \\
& \mathrm{q}_{3}=\bar{q}_{3} / \alpha-\beta \overline{\mathrm{p}}_{3} / \alpha-\mathrm{A}_{\psi}-O(\varepsilon) \text { and } \\
& \mathrm{H}=(1 / 2)\left\{\mathrm{B}\left(\overline{\mathrm{p}}_{3}^{2}+\overline{\mathrm{q}}_{3}^{2}\right)+\mathrm{R}\left(\varepsilon \overline{\mathrm{p}}_{1}-\varepsilon \overline{\mathrm{q}}_{2}\right)^{2}\right\}+O(\varepsilon)
\end{aligned}
$$

If we iterate the transformation we obtain

$$
H=H_{N}\left(\varepsilon p_{1}, q_{1}, \varepsilon p_{2}, \varepsilon q_{2}, p_{3}^{2}+q_{3}^{2}, \varepsilon\right)+O\left(\varepsilon^{N}\right)
$$

so that we may take $2 \mu=p_{3}^{2}+q_{3}^{2}$ and we find 


$$
\begin{aligned}
H= & \mu B\left(\varepsilon p_{2}, \varepsilon q_{2}\right)+(1 / 2) R\left(\varepsilon p_{2}, \varepsilon q_{2}\right) \cdot\left(\varepsilon p_{1}-\varepsilon q_{2}\right) \\
& \left.+\varepsilon H_{1}\left(\varepsilon p_{1}, q_{1}, \varepsilon p_{2}, \varepsilon q_{2}, \mu, \varepsilon\right)+O(\varepsilon)^{N}\right)
\end{aligned}
$$

and for the most part we need only $\mathrm{H}_{0}$.

We may readily construct the second or longitudinal adiabatic invariant after we note that in $(49)\left(\varepsilon p_{1}, q_{1}\right)$ vary on the $O(1 / \varepsilon)$ scale while $\left(\varepsilon p_{2}, \varepsilon q_{2}\right)$ vary on the $O\left(1 / \varepsilon^{2}\right)$ time scale. We, have no difficulties with periodicity or resonance problems as $\mathrm{H}$ and the orbits in the $\left(\varepsilon p_{1}, q_{1}\right)$ plane are clearly periodic of period $2 \pi$ in $q_{1}$. An argument parallel to that given in the previous section shows the existence of the second adiabatic invariant

$$
J=(1 / 2 \pi): \int_{0}^{2 \pi} \varepsilon p_{1} d q_{1}+O(\varepsilon)=\varepsilon p_{1}+O(\varepsilon)
$$

Clearly in the axisymmetric case the adiabatic invariant $J$ becomes the exact constant of the motion $p_{1}$. The Hamiltonian for the remaining drift motion then becomes

$$
H=\mu B\left(\varepsilon p_{1}, \varepsilon q_{2}\right)+(1 / 2) R\left(\varepsilon \cdot p_{2}, \varepsilon q_{2}\right)\left(J-\varepsilon q_{2}\right)^{2}+O(\varepsilon)
$$

Since $H=$ const. on the orbit we may identify the drift orbit, to leading order in $\varepsilon$ as

$$
\mathrm{H}_{0}=\mu \mathrm{B}(\lambda, \psi)+(1 / 2) \mathrm{R}(\lambda, \psi) \cdot(\mathrm{J}-\lambda)^{2}=\text { const. }
$$

and we may map out the drift surfaces from (52)in the $\psi, \lambda$ plane. We see that the drift surfaces are not localized on a surface $\psi=$ const.. but particles may drift far across such surfaces. These drifts occur in the axisymmtric case. The principal effect of the non-axisymmetric 
fields is to reconnect the orbits through the critical points $0=\mathrm{H}_{0, \lambda}$ $=\mathrm{H}_{0, \psi}$. We see that the orbits in this case are quite different from those of the preceding section. 


\section{An Intermediate Guiding Center Expansiọn}

The results of the two previous sections in which we took $B \sim 1$ but ${ }^{\mathrm{B}} \mathrm{p} \mathrm{l}$ or $\mathrm{B}_{\mathrm{p}} \sim \varepsilon$ are so dissimilar that it is of interest to consider an intermediate case. In this section we take $\mathrm{B}_{\mathrm{T}} \sim 1, \mathrm{~B}_{\mathrm{p}} \sim \vee \varepsilon$ are $B_{\mathrm{NA}} \sim \varepsilon$. Such an ordering may well apply to many Tokamak devices in which $\mathrm{B}_{\mathrm{p}} / \mathrm{B}_{\mathrm{T}}<1$ but it may not be quite as smail as $O(\varepsilon)$. We readily develop the magnetic moment adiabatic invariant series and the associated guiding oenter Hamiltonian. We need the quiding center Hamiltonian correct through $O(\varepsilon)$ and we give most of the details of this development in the Appendix. We find, however, that there is no second adiabatic invariant. The structure of the second invariant is so different in the two cases just considered that it should be no surprise that none exists in the transitional case studied here. We examine the question with two distinct sets of canonically conjugate variables. With the first set, similar to the variables of Sec. III, we find what looks like a second adiabatic invariant, but a careful examination indicates that the "irvariant" is constant nver a large but limited time interval which is too small to see particle drifts. With the second set of variables, similar to those of Sec. IV, another picture appears. The Hamiltonian ontains certain variables which have a rapid oscillation of smill amplitude. Thus, no second invariant is possible. But for this situation we present a more or less conventional perturbation expansion of the trapped particle orbits and we determine the flux surface on which the particle lies as a function of time. We show that there is a change in the flux surface at the two lips of the trapper orbit and we give a formula for the shift. We also 
study the net change in the flux surface after passing through many orbit tips and we examine some resonant and non-resonant phenomena. The passing particles are much simpler to treat and we also comment briefly on their drifts across the flux surfaces.

We again modify the $\theta$ component of the vector potential $\mathrm{A}^{\prime},(8)$

$$
\lambda_{\dot{\theta}}^{\prime}=\psi(\varepsilon r, \varepsilon \tau) /\left(\varepsilon^{3 / 2} r^{\prime}\right)
$$

so that $\mathrm{B}_{\mathrm{T}}$ is unaffected, but

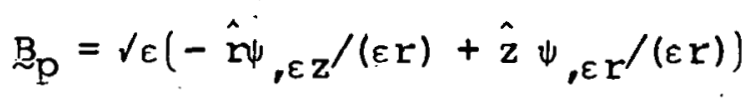

and

$$
\begin{aligned}
H= & (1 / 2)\left\{\left(P_{r}-\lambda \psi_{,} r / \varepsilon-A_{r}\right)^{2}+\left(P_{z}-\lambda{ }_{,}, \varepsilon z^{/ \varepsilon-A_{z}}\right)^{2}\right. \\
& \left.+\left(\varepsilon P_{2}-\psi / \gamma \varepsilon\right)^{2} /(\varepsilon r)^{2}\right\} .
\end{aligned}
$$

We introduce the gererator

$$
F=\left(\psi / \varepsilon-\sqrt{ } \varepsilon p_{1}\right) p_{2}+\lambda p_{3} / \varepsilon-p_{2} p_{3}+p_{1} \theta
$$

so that

$$
\begin{aligned}
& \psi=\varepsilon^{3 / 2} p_{1}+\varepsilon q_{2}+\varepsilon p_{3} \\
& \dot{\lambda}=\varepsilon p_{2}+\varepsilon q_{3} \\
& \theta=q_{1}+\downarrow \varepsilon p_{2}
\end{aligned}
$$

and (43) holds for the connection between new and old momenta. If we introduce $E, F, G, R, A_{\psi}$ and $A_{\lambda}$ according to (45) but replace $\psi, \lambda, \theta$ by (54) then we find

$$
H=(1 / 2)\left(E\left(q_{3}+A_{\psi}\right)^{2}+2 F\left(q_{3}+A_{\psi}\right)\left(p_{3}-A_{\lambda}\right)+G\left(p_{3}-A_{\lambda}\right)^{2}\right)
$$




$$
+(1 / 2) R\left(v \varepsilon q_{2}+\sqrt{ } \varepsilon p_{3}\right)^{2}
$$

We see that the scaling of our variables is now $\varepsilon^{3 / 2} p_{1}=O(1), \varepsilon p_{2}=$ $O(1), \sqrt{ } \varepsilon q_{2}=O(1)$, and both $p_{3}, q_{3}=O(1)$. We have not scaled $q_{1}$, but since $A_{\psi}, A_{\lambda}$ are periodic of period $2 \pi$ in $\theta$ and $q_{1}$, the magnitude of $q_{1}$ does not affect the other variables. We now have

$$
H=H\left(\varepsilon^{3 / 2} \dot{p}_{1}, q_{1}, \varepsilon \dot{p}_{2}, v \varepsilon q_{2}, p_{3}, q_{3}, \varepsilon\right)
$$

and both $\left(\varepsilon^{3 / 2} p_{1}, q_{1}\right)$ and $\left(\varepsilon p_{2}, v \varepsilon q_{2}\right)$ are on the same, slow, $O\left(\varepsilon^{-3 / 2}\right)$ time scale while $\left(p_{3}, q_{3}\right)$ are on the $O(1)$ time scale.

In the appendix we present the details of the canonical transformations which introduce the magnetic monent adiabatic invariant and we show that the transformed Hamiltonian has the structure

$$
\begin{aligned}
H= & H_{0}\left(\psi, \lambda, V \varepsilon{\hat{q_{2}}}_{2, \varepsilon}\right)+\varepsilon \dot{H}_{2}\left(\psi, \lambda, V \varepsilon \hat{\grave{q}}_{2}, \theta, \varepsilon\right) \\
& +\varepsilon^{3 / 2} \mathrm{H}_{3}\left(\psi, \lambda, v \varepsilon \hat{q}_{2}, \theta, \varepsilon\right)+\ldots .
\end{aligned}
$$

where $\psi, \lambda, \theta$ are replaced according to $(\mathrm{A} 22)$ and we defire

$$
\begin{aligned}
& H_{0}=\mu(B+\varepsilon R E /(2 B))+(1 / 2)\left(V^{\prime} \varepsilon \hat{q}_{2}\right)^{2}\left(R-\varepsilon R^{2} E / B\right) \\
& -\varepsilon R\left(V \varepsilon \hat{a}_{2}\right)\left\langle A_{\lambda} A_{i, j, \theta}\right\rangle \\
& H_{2}=\mu B^{2}\left(\left(A_{\psi} / B\right), \lambda-\left(A_{\lambda} / B\right), \psi\right) ;
\end{aligned}
$$

and according to (A 23) we may consider the coordinate of the flux surface on which the particle moves to be $\varepsilon^{3 / 2} \hat{p}_{1}+\varepsilon \hat{q}_{2}$. We have scaled time according to the period for a cyclotron oscillation. Since we have eliminated this oscillation from the Hamiltonian it is 
convenient to rescale time and the variables according to the drift time scale, which as we just saw is $O\left(\varepsilon^{-3 / 2}\right)$. If we set $\tau=\varepsilon^{3 / 2} t$ we see tha

$$
\begin{aligned}
\hat{p}_{1} d \hat{q}_{1} & +\hat{p}_{2} d \hat{q}_{2}-H d t \\
= & \varepsilon^{-3 / 2}\left(\left(\varepsilon^{3 / 2} \hat{p}_{1}\right) d \hat{q}_{1}+\left(\varepsilon \cdot \hat{p}_{2}\right) d\left(\sqrt{\varepsilon} \hat{q}_{2}\right)-H d \tau\right),
\end{aligned}
$$

so that a resealing canunlcal transtormation would he $3 / 2 \ddot{p}_{1}=p_{1}$, $\hat{q}_{2}=q_{1}, \varepsilon \hat{p}_{2}=p_{2}, v \varepsilon \hat{q}_{2}=q_{2}$, and

$\tau=\varepsilon^{3 / 2}$ t and with this transformation and the definition

$$
\begin{gathered}
\eta=\sqrt{ }, \\
\dot{H}=H_{0}\left(\psi, \lambda, q_{2}, \varepsilon\right)+{ }^{2}{ }^{2} H_{2}\left(\psi, \lambda, q_{2}, \theta, \varepsilon\right)+{ }^{3}{ }^{3} H_{3}\left(\psi, \lambda, q_{2}, \theta, \varepsilon\right)+\ldots
\end{gathered}
$$

where $\mathrm{H}_{0}$ and $\mathrm{H}_{2}$ are still given by (57), but

$$
\begin{aligned}
& \psi=p_{1}+n q_{2} \\
& \lambda=p_{2} \\
& H=q_{1}+p_{2} / n
\end{aligned}
$$

We see that the second and higher order terms in the Hamiltonian have rapid dependence on the canonical variable $p_{2}$. It is this rapid dependence on $p_{2}$ that destroys the possibility of the existence of an adiabatic invariant.

It is instructive to study the possibility of the existence of a second adiabatic invariant before we proceed with a direct analysis 
of (59), (60). To this end we go back to the Hamiltonian (56) and variables (A 23). The question becomes clearer after yet another canonical transformation. If we introduce the variables

$$
\begin{aligned}
& \varepsilon P_{2}=-\sqrt{\varepsilon} \hat{q}_{2} \\
& Q_{1}=\hat{q}_{1}+\sqrt{\varepsilon} \hat{p}_{2}
\end{aligned}
$$

$$
\begin{aligned}
& \varepsilon \mathrm{p}_{2}=-\sqrt{ } \varepsilon \hat{\mathrm{q}}_{1} \\
& \varepsilon \hat{\mathrm{o}}_{2}=\varepsilon^{3 / 2} \hat{\mathrm{p}}_{1}+\varepsilon \hat{\mathrm{q}}_{2}
\end{aligned}
$$

then a direct computation of Poisson brackets shows that

$$
\left\{P_{i}, o_{j}\right\}=\delta_{i j},\left\{P_{1}, P_{2}\right\}=\left\{o_{i}, Q_{j}\right\}=0 \text {, so that the }
$$

transformation is indeed canonical, (7) and the Hamiltonian is given by

$$
\begin{aligned}
H= & H_{0}\left(\psi, \lambda,-\varepsilon P_{1}, \theta, \varepsilon\right)+\varepsilon H_{2}\left(\psi, \lambda,-\varepsilon P_{1}, \theta, \varepsilon\right) \\
& +\varepsilon^{3 / 2} H\left(\psi, \lambda,-\varepsilon P_{1}, \theta, \varepsilon\right)+\ldots
\end{aligned}
$$

with the substitutions

$$
\begin{aligned}
& \psi=\varepsilon Q_{2} \\
& \lambda=\varepsilon P_{2}+\sqrt{\varepsilon} O_{1} \\
& \theta=Q_{1}
\end{aligned}
$$

and with $\mathrm{H}_{0}, \mathrm{H}_{2}, \mathrm{H}_{3}$, etc. still given by (57). Parenthetically we note that the variables (63) are essentially the same as those of 
section IV, compare (42) and (53), while the variables used elsewhere in this section are essentially those of section III, compare (16) and (A 22). With the Hamiltonian (62) we see that $\left(P_{1}, O_{1}\right)$ vary on the $O(1 / \varepsilon)$ time scale, but $\left(P_{2}, Q_{2}\right)$ vary on the $O\left(1 / \varepsilon^{2}\right)$ time scale. Thus we have the first prerequisite for the construction of an adiabatic invariant, the separation of time scales. We note that the variablea we use for this discussion destroy the axisymmetry of motion in an axisymetric magnetic field.

In order to proceed with the construction of the adiabatic invariant, we must also find families of periodic orbits. If we examine the leading order Hamiltonian $\mathrm{H}_{0}$ in (63) we see that the variable $\lambda=\varepsilon \mathrm{P}_{2}+\sqrt{\varepsilon} \theta$ occurs. Thus, an orbit does not close on itself until $\sqrt{ } \varepsilon \theta$ is $O(1)$, or at least $\tau \sim 1 / \varepsilon^{3 / 2}$. Even over such a lung time Interval there will not be a family of periodic orbits but only isolated periodic orbits. Clearly we are back to the study of resonance problems explored at the end of section III.We might view the question in another way. We could expand a function

$$
f(\lambda)=f\left(\varepsilon P_{2}+\sqrt{ } \varepsilon \theta\right)=f\left(\varepsilon P_{2}\right)+\sqrt{ } \varepsilon \theta f^{\prime}\left(\varepsilon P_{2}\right)+\ldots \text {. In this way the }
$$
lowest order Hamiltonian would be independent of $Q_{1} \equiv \theta$, and so the orbits would be periodic on an $O(1)$ time scale. We could then easily construct a second adiabatic invariant exactly as in section IV ard we would have particles confined on or near flux surfaces. Apparently a second invariant does exist, but let us consider the matter in greater detail. For the expansion just given to hold we require $\sqrt{ } \varepsilon \theta$ must be smal1. When $\sqrt{ } \varepsilon \theta$ is $O(1)$ the representation of the Hamiltonian is poor. Thus, we obtain a second adiabatic invariant, but only over the limited time interval $t \leqslant \varepsilon^{-3 / 2}$. for which $\sqrt{ } \theta \theta$ is $\leqslant 1$. Over this 
interval we might see what looks like an adiabatic invariant, but as time increases, the constancy must fail. We see shortly that the drifts do indeed occur, but on an $O\left(\varepsilon^{-11 / 4}\right)$ time scale, a result fully consistent with the anarlysis here. We set aside the possibility of an adiabatic invariant as the invariant we found cannot describe the orbit for a long enough time and we return to the variables and Hamiltonian given by (59) and (60).

Hamilton's equations for the system (59); (60) are

$$
\begin{aligned}
& -\dot{\mathrm{p}}_{1}=n^{2} \mathrm{H}_{2, \mathrm{q}_{1}}+n^{3} \mathrm{H}_{3, \mathrm{q}_{1}}+\ldots, \quad \dot{\mathrm{q}}_{1}=\mathrm{H}_{0, \mathrm{p}_{1}}-n^{2} \mathrm{H}_{2, \mathrm{p}_{1}}-n^{3} \mathrm{H}_{3, \mathrm{p}_{1}}+\ldots \\
& -\dot{p}_{2}=\mathrm{H}_{0, q_{2}}+n^{2} \mathrm{H}_{2, \mathrm{q}_{2}}+n^{3} \mathrm{H}_{3} \mathrm{q}_{2}+\ldots, \quad \dot{q}_{2}=\mathrm{H}_{0, \mathrm{p}_{2}}+n \mathrm{H}_{2, \mathrm{q}_{1}}+n^{2}\left(\mathrm{H}_{3, \mathrm{q}_{1}}+\mathrm{H}_{2, \mathrm{p}_{2}}\right)+\ldots
\end{aligned}
$$

where we consider $H=H\left(p_{1}+n q_{2}, p_{2}, q_{2}, q_{1}+p_{2} / n\right)$ and by convention $H, p_{2}$ means a derivative with respect to the second argument only. That part of a partial derivative of $H$ with respect to $\mathrm{p}_{2}$ coming from differentiation with respect to the fourth argument is represented as a derivative with respect to $q_{1}$. With this convention each term in (64) is of the order of magnitude in $n$ explicitly exhibited. We intend to study the drift motion by examining (64.) over a limited time interval. and then repeating the process for the next limited time interval. With such an approach we may employ a conventional perturbation expansion in powers of $n$ in (64). We expand in the form

$$
\begin{aligned}
\left(p_{i}(t), q_{i}(t)\right)= & \left(p_{1}^{(0)}(t, n), q_{1}^{(0)}(t, n)\right) \\
& +n\left(p_{1}^{(1)}(t, n), q_{1}^{(1)}(t, n)\right)+n^{2}\left(p_{1}^{(2)}(t, n), q_{1}^{(2)}(t, n)\right)+\ldots
\end{aligned}
$$

where terms $\left(p_{1}^{(j)}(t, n), q_{1}^{(i)}(t, n)\right)$ may be $o(1)$ : in $n$ although they art never larger than $O(1)$. To lowest order we find 


$$
\dot{p}_{2}^{(2)}=\mathrm{H}_{0, q_{2}}^{(0)}, \quad \dot{q}_{2}^{(2)}=\mathrm{H}_{\delta}^{(0)}, \underline{p}_{2}
$$

We may solve the oystem (65) quite simply since $\mathrm{p}(0)=$ const. and $q_{1}$ doc3 not appear in $\mathrm{H}_{0}$. The motion reduces in the $p_{2}, q_{2}$ plane to the trajectory $\mathrm{H}_{0}\left(p_{1}^{(0)}+\varepsilon q_{1}^{(0)}, p_{2}^{(0)}, q_{2}^{(0)}\right)=$ const. We may again distinguish two classes of orbits, closed curves in the $p_{2}, q_{2}$ plane corresponding to trapped particle orbits, and unbounded curves corresponding to passing particles. The passing particles generate a periodic orbit when one recognizes that according to (34) when $\lambda \equiv \mathrm{p}_{2}$ increases by $\Lambda(\psi)$ the point $(\psi, \lambda, \theta)$ returns to the same physical point. We examine trapped particles in greatest detail, although we give some indication of what we believe is the solution with passing particles.

In the solution of the full system (64) by perturbation techniques based on the lowest order system (65) we must always solve the variational equations associated with the lowest order system. If we hold $p_{1}$ constant the variational system is a linear set of differential equations for $p_{2}(t), q_{2}(t)$ :

$$
\begin{aligned}
& -\dot{p}_{2}(t)=H_{\delta, q_{2} p_{2}}^{(0)} p_{2}(t)+H_{, q_{2} q_{2}}^{(0)} q_{2}(t)+f(t) \\
& \dot{q}_{2}(t)=H_{0}(0) p_{2} p_{2} p_{2}(t)+H_{,}{ }_{, q_{2} p_{2}}^{(0)} q_{2}(t)+g(t),
\end{aligned}
$$

where such a term as $\mathrm{H}_{0}\left({ }_{1}\right)_{2} \mathrm{q}_{2} \mathrm{p}_{2}$ is evaluated at the zeroth order solution of (65). One solution of the homogeneous system associated with (66) 
is $\left(\dot{p}_{2}^{(0)}(t), \dot{q}_{2}^{(0)}(t)\right)$ which we denote by $(P(t), Q(t))$. We introduce a second solution $(p(t), q(t))$ normalized so that

$$
p(t) q(t)-Q(t) p(t)=1
$$

and the solution to the variational system (66) is

$$
\begin{aligned}
& p_{2}(t)=P(t) I_{1}(t)+p(t) I_{2}(t) \\
& q_{2}(t)=Q(t) I_{1}(t)+q(t) I_{2}(t)
\end{aligned}
$$

where

$$
I_{1}=-f_{f}^{t} d t^{\prime}\left(f\left(t^{\prime}\right) \dot{q}\left(t^{\prime}\right)+g\left(t^{\prime}\right) p\left(t^{\prime}\right)\right)
$$

and

$$
I_{2}=\int_{0}^{t} d t^{\prime}\left(f\left(t^{\prime}\right) Q^{\prime}\left(t^{\prime}\right)+g\left(t^{\prime}\right) P\left(t^{\prime}\right)\right)
$$

The first order solution satisfies the system

$$
\begin{aligned}
& -\dot{p}(1)=n H_{2, q_{1}}^{(0)}+n \dot{H}_{2}(0) q_{1} q_{1}(p(l) / n) \text {. }
\end{aligned}
$$

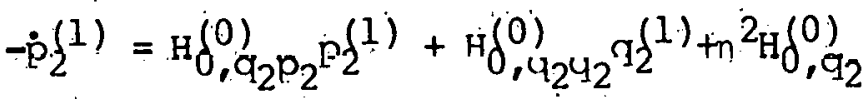

$$
\begin{aligned}
& \dot{q}_{2}^{(1)}=\mathrm{H}_{0}(0) p_{2} \mathrm{p}_{2} \mathrm{p}_{2}^{(1)}+\mathrm{H}_{0}(0) \mathrm{p}_{2} \mathrm{q}_{2} \mathrm{q}_{2}^{(1)}
\end{aligned}
$$

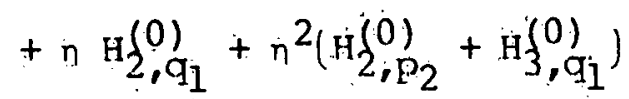

The formulas $(66)-(70)$ apply equalily well to trapped or untrapped particles.

We now turn our attention specifically to trapped particles and estimate the solution to (70) appropriately for trapped particles. We readily find, following the notation of (68), that 


$$
I_{1}=-n \int_{0}^{t} d t^{\prime} H_{2, q_{1}}^{(0)}\left(t^{\prime}\right) p\left(t^{\prime}\right)+o\left(n^{2}\right)
$$

and

$$
I_{2}=o\left(n^{2}\right)
$$

so that

$$
\begin{aligned}
-p_{1}^{(1)}(t)= & n \int_{0}^{t} d t^{\prime} H_{2}^{(0), q_{1}}\left(t^{\prime}\right) \\
& -11 f_{0}^{t} a t^{\prime} H_{2}^{(0)}, q_{1} q_{1}\left(t^{\prime}\right) P\left(t^{\prime}\right) \cdot \int_{0}^{t^{\prime}} a t^{i n} H_{2, q_{1}}^{(0)}\left(t^{\prime \prime}\right) p\left(t^{\prime \prime}\right)+n\left(\eta^{2}\right) .
\end{aligned}
$$

From the identity

$$
n(d / d t) H_{2}(0), q_{1}=H_{0}(0), q_{1} q_{1} P(t)+O(n)
$$

we readily conclude

$$
-p f^{1)}=f^{\prime} d t^{\prime \prime} H_{, q_{I}}^{(0)}(t ") p\left(t^{\prime \prime}\right)+o\left(n^{2}\right)
$$

so that the shift in the flux surface as a function of time is

$$
\begin{aligned}
\Delta \psi(t)= & -\eta^{2} f_{0}^{t} d t^{\prime} H_{2, q_{1}}^{(0)}\left(t^{\prime}\right) \\
& -n^{2} O(t) f_{0}^{t} d t^{\prime} H_{2 ;}(0) q_{1}\left(t^{\prime}\right) p(t)+o\left(n^{3}\right) .
\end{aligned}
$$

We have not yet specified the point $t=0$ or the time interval over which we svaluate $\Delta \psi(t)$. We obtain a particularly simple and symnetric representation if we set $t=0$ to be the point at which $\dot{q}_{2}^{(0)}(t)=0$, or $Q(t=0)=0$. We let $T(\psi)$ be the period for this orbit and we then find

$$
\Delta \psi(T)=-n^{2} f^{T} d t^{\prime} H_{2}{ }_{2}^{0} q_{1}\left(t^{\prime}\right)+o\left(n^{3}\right) .
$$


The trapped particle orbit $\left(p f^{(0)}(t), q_{2}^{(0)}(t)\right)$ is a closed curve in the $\left(p_{2}, q_{2}\right)$ plane. We define the tips of the orbit as the points at which $p_{2}(t) \equiv \lambda$ assumes the largest and smallest values. Clearly these are points at which $P(t)$ vanishes. Equally, the points of stationary $V_{\|} \equiv q_{2}(t)$ are the points at which $Q(t)=0$. During one period the toroidal angle $\theta$ increases by an amount

$$
\Delta \theta=\int_{0}^{T(\psi)} \mathrm{dt} \mathrm{H}_{0}^{\mathrm{O}, \mathrm{P}_{1}}
$$

Let $t_{1}$ and $t_{2}$ be the times of the two consecutive banana tips, $0<t_{1}<$ $t_{2}<T$, so that by symmetry

$$
q_{1}^{(0)}\left(t_{2}\right)-q_{1}^{(0)}\left(t_{1}\right)=\Delta \theta / 2
$$

Let us examine the essential element in (71)

$$
I=\int_{0}^{T} d t H_{2,} q_{1}\left(p_{1}^{(0)}+n q_{2}^{(0)}(t), p_{2}^{(0)}(t), q_{2}^{(0)}(t), q_{1}^{(0)}(t)+p_{2}^{(0)}(t) / n\right) \text {, }
$$

Except in the neighborhood of the points $t_{1}, t_{2}$ where $P(t)=0$ we may use

$$
\mathrm{H}_{2, \mathrm{q}_{1}}=n\left((\mathrm{~d} / \mathrm{dt}) \mathrm{H}_{2}\right) / \mathrm{P}(\mathrm{t})+\mathrm{O}(\mathrm{n})
$$

and integrate by parts to conclude that $I=O(n)$. Hence, the leading order terms in (7.3) rome entirely from the stationary points of the integrand $t_{1}$ and $t_{2}$. We are led to perform a stationary phase evaluation of (73). If we expand about an orbit tip $t=t_{i}, i=1,2$ we find

$$
p_{2}^{(0)}(t)=p_{2}^{(0)}\left(t_{i}\right)+\left(\ddot{p}_{2}^{(0)}\left(t_{i}\right) / 2\right)\left(t-t_{i}\right)^{2}+\ldots \text { and if we }
$$

introduce 


$$
C_{i}=p_{2}^{\prime \prime}(0)\left(t_{i}\right)=-H_{, q_{2} q_{2}}^{(0)}\left(t_{i}\right) \underset{{ }_{r}}{p_{2}}\left(t_{i}\right),
$$

then we readily find

$$
\begin{aligned}
& \Delta \psi=-n^{5 / 2} \sqrt{2} \int_{0}^{\infty}(d x / v x) \cdot\left\{H_{2}^{(0)} q_{1}\left(\psi(t), \lambda\left(t_{1}\right), q_{2}^{(0)}\left(t_{1}\right), \theta+p_{2}^{(0)}\left(t_{1}\right) / n+x C_{1}\right)\right. \\
& \left.+\mathrm{H}_{2}(0) \mathrm{q}_{1}\left(\psi(t), \lambda\left(t_{2}\right), \mathrm{q}_{2}^{(0)}\left(t_{2}\right), \theta+\Delta \theta / 2+\mathrm{p}_{2}^{(0)}\left(t_{2}\right) / n+\mathrm{xC}_{2}\right)\right\}+o\left(\eta^{3}\right)
\end{aligned}
$$

and

$$
\Delta \theta=\int_{0}^{T}(\psi) \text { dt } \mathrm{H}_{0}(0), \mathrm{P}_{1}
$$

In the evaluation of $(75), \mathrm{H}_{0}$ and the orbits $\mathrm{p}_{2}^{(0)}(t), \mathrm{q}_{2}^{(0)}(t)$ are held fixed, so that (75) is a property of the orbit alone. Further, the orbit is characterized by the values of $\psi$ and of $H$ (a constant) alone. Thus, one may view (75) as a discrete process which describes the slow drift of the trapped particle orbits

$$
\begin{aligned}
& \psi_{n+1}-\psi_{n}=n^{5 / 2} f\left(\psi_{n}, \theta_{n}\right) \\
& { }_{n+1}=\theta_{n}=g\left(\psi_{n}\right)
\end{aligned}
$$

where $f$ and $g$ are given by (75). The asymptotic evaluation of (71) as (75) is identical with the usual stationary phase integration for $H_{2}\left(\psi, \lambda, q_{2}, \theta\right)=f\left(\psi, \lambda, q_{2}\right) \exp ($ in $\theta), n \neq 0$. We see from (75) that only the neighborhoods of the trapped particle orbit tips contribute in leading order to $\Delta \psi$. The existence of a second adiabatic invariant 
would imply that particle drift is a continuous process rather than a discrete one. Thus, (75) is inconsistent with the existence of an adiabatic invariant as comonly understood.

We may show resonance phenomena in the system (75), (76). We examine (76) after $\mathrm{N}$ steps where we assume $\mathrm{Nn}^{5 / 2}<1$ and we find

$$
\psi_{N+1}-\psi_{1}=n^{5 / 2}\left(\sum_{n=0}^{N-1} f\left(\psi_{1},{ }^{\theta} 1^{+n g}\left(\psi_{1}\right)\right)+o(1)\right)
$$

If $g\left(\psi_{1}\right)$ is a rational multiple of $2 \pi$ then $\psi_{N+1}-\psi_{1}$ is proportional to $N$, while for $g\left(\psi_{1}\right)$ an irrational multiple of $2 \pi, \psi_{N+1}-\psi_{1}$ is proportional to $\mathrm{Nn}^{5 / 2}\left\langle f\left(\psi_{1}, \theta\right)\right\rangle$, which in our case vanishes. Since the process (76) is only accurate to a correction $O\left(n^{7 / 2}\right)$, we may approximate any irrational with a rational with an error, say $0(\checkmark n)$, and obtain a mean shift $\Delta \psi$. If $f(\psi, \theta)$ contains only one or at most a few harmonics, then the resonance condition is far more stringent and our simple model may not suffice. In this case $g\left(\psi_{1}\right)$ must be well approximated by a rational with a denominator equal to one of the harmniss present in the fourier series representation of $f(\psi, \theta)$. otherwise the drift approximately vanishes. When we consider the full $\psi$ dependence in (76) rather than the approximation (77), the process becomes more intricate and the posibility of slow drift across flux surfaces occurs.

There is still another mechanism of significant silow drift across flux surface beyond those implied by (7.6). In many cases the net effect of the discrete process (76.) will give a net zero drift, especially when $\langle f\rangle=0$ or there are only a very few harmonics in the Fourier series expansion of $f(\psi, \theta)$. It is easy to show however that if 
one carries the expansion in (71) through fourth order in $n$; then there are terms in $\Delta \psi$ which do not average to zero and which do give rise to a small but steady $\Delta \psi$ which is $O\left(n^{4}\right)$. The actual evaluation of the drift is an exceedingly laborious calculation and we do not have the result. It should be noted that when the full $\psi$ dependence of (76) is used, similar terms $O\left(n^{4}\right)$ appear in the disckete process as well. In the strongly resonant nase we do mot nood thic cffcct to deseribe drifts, but off resonance such terms do produce a determinable drift motion. We are not, however, prepared to explore the matter further.

For the case of passing particles (56) - (71) still hold, but now $P(t)=p_{2}^{(0)}(t)$ never vanishes so that there is no stationary phase point in the evaluation of (71). Although $\mathrm{p}_{2}^{(0)}(\mathrm{t})$ is not periodic, $p f^{0)}(t)-t \Lambda(\psi)$ is periodic so that $P(t)$ is periodic and we find from (71)

$$
\Delta \psi(T)=o\left(n^{3}\right)
$$

For passing particles there is no discrete process such as (76), although if we extend (71) to higher order in $n$ we find that there is, just as for trapped particles, a higher order small drift which does not average to zero. Its calculation is an intricate matter and we are unprepared to offer detailed results; it is easy to show, however, that $\Delta \psi(T)$ in this case is $o\left(n^{4}\right)$, so that the drifts are smallex than for trapped particles. 
Appendix A. The Intermediate Scaling Guiding Center Hamiltonian

In Section $V$ we require the Hamiltonian to more than lowest order. Here we give the sequence of canonical transformations leading to the desired form. This appendix gives an explicit example of how we may employ canonical transformations to simplify the problem and obtain higher order corrections in the Hamiltonian. We start from the Hamiltonian (55), with $E, F, G, R, A_{\psi}$ and $A_{\lambda}$ defined by (45) and with the space coordinates $\psi, \lambda, \dot{\theta}$ replaced by (54). Throughout this treatment we shall onsider functions of space coordinates as functions of $\psi, \lambda, \theta$ followed by some replacements of $\psi, \lambda, \theta$ by canonicaliy conjugate onordinates. We start by expanding the various functions of the space coordinate $\psi, \lambda, \theta$ in Tayior series in $\varepsilon p_{3}, \varepsilon \dot{q}_{3}$ in the form

$$
\begin{aligned}
& f(\psi, \lambda, \theta)=f\left(\varepsilon^{3 / 2} \dot{p}_{1}+\varepsilon q_{2}+\varepsilon p_{3}, \varepsilon p_{2}+\varepsilon q_{3}, q_{1}+j \varepsilon \dot{p}_{2}\right) \\
& =f\left(\varepsilon^{3 / 2} p_{1}+\varepsilon q_{2} ; \varepsilon p_{2}, q_{1}+\gamma \varepsilon p_{2}\right) \\
& +\varepsilon\left(\mathrm{f}, \psi \mathrm{p}_{3}+\mathrm{f}, \lambda \mathrm{q}_{3}\right)+\left(\varepsilon^{2} / 2\right)\left(\mathrm{f}, \psi \psi \mathrm{p}_{3}^{2}+2 \mathrm{f}, \psi \lambda \mathrm{p}_{3} \mathrm{q}_{3}+\mathrm{f}, \lambda \lambda q_{3}^{2}\right)+\ldots
\end{aligned}
$$

where $\psi, \lambda, \theta$ on the right hand side are replaced by $\psi=\varepsilon^{3 / 2} \dot{p}_{1}+\varepsilon q_{2}$, $\lambda \doteq \varepsilon p_{2}$, ầi $\dot{\theta} \equiv q_{1}+\sqrt{ } \mathrm{q}_{2} \cdot$. We nö start the canonical transformations with the generator

$$
\begin{aligned}
F= & \left(\bar{p}_{3}+A_{\lambda}\left(\varepsilon{ }^{3 / 2} \bar{p}_{1}+\varepsilon q_{2} ; \varepsilon \bar{p}_{2}, q_{1}+\varepsilon \bar{p}_{2}\right)\right) \\
& +\left(q_{3}+A_{j j}\left(\varepsilon^{3 / 2} \dot{\bar{p}}_{1}+\varepsilon q_{2} ; \varepsilon \bar{p}_{2}, q_{1}+\varepsilon \bar{p}_{2}\right)\right)+\overline{\bar{q}}_{1} q_{1}+\overline{\bar{p}}_{2} q_{2},
\end{aligned}
$$


$-50-$

$$
\begin{aligned}
& q_{1}=\bar{q}_{1}+o\left(\varepsilon^{3 / 2}\right), \quad p_{1}=\bar{p}_{1}+o(1) \\
& \bar{p}_{3}=p_{3}-A_{\lambda}\left(\varepsilon^{3 / 2} p_{1}+\varepsilon q_{2}, \varepsilon p_{2}, q_{1}+v \varepsilon p_{2}\right)+o\left(\varepsilon{ }^{3 / 2}\right) \\
& \bar{q}_{3}=q_{3}+A_{\psi}\left(\varepsilon^{3 / 2} p_{1}+\varepsilon q_{2}, \varepsilon p_{2}, q_{1}+v \varepsilon p_{2}\right)+o\left(\varepsilon^{3 / 2}\right) \\
& p_{2}=\bar{q}_{2}+o(\varepsilon) \\
& \bar{q}_{2}=q_{2}+v \varepsilon\left(A_{\lambda, \theta} \bar{q}_{3}+A_{\psi, \theta} \bar{p}_{3}+A_{\psi, \theta} A_{\lambda}\right)+o(\varepsilon)
\end{aligned}
$$

where we may evaluate the potentials at the new or old coordinates. Since we wish to follow the flux surface on which a particle lies we must determine the combination $\varepsilon^{3 / 2} p_{1}+\varepsilon q_{2}$ with some care. We see that

$$
\varepsilon^{3 / 2} \bar{p}_{1}+\varepsilon \bar{q}_{2}=\varepsilon^{3 / 2} p_{1}+\varepsilon q_{2}+o\left(\varepsilon^{2}\right)
$$

and that the error term is just a point function of the coordinates, Independent of time and not equivalent to a particle drift across a flux surface. After the transformations

$$
\begin{aligned}
H & =(1 / 2)\left\{\left(E \overline{\mathrm{q}}_{3}^{2}+2 F \overline{\mathrm{q}}_{3} \overline{\mathrm{p}}_{3}+\mathrm{G} \overline{\mathrm{p}}_{3}^{2}\right)\right. \\
& \left.+\mathrm{R}\left\{\sqrt{ } \varepsilon\left(\overline{\mathrm{q}}_{2}+\mathrm{A}_{\lambda}\right)\right\}^{2}\right\}+\sqrt{\varepsilon} \overline{\mathrm{p}}_{3} \mathrm{R}^{\prime} \varepsilon\left(\overline{\mathrm{q}}_{2}+\mathrm{A}_{\lambda}\right) \\
& +\varepsilon \mathrm{T}_{1} / 2+\mathrm{O}\left(\mathrm{F}^{3 / 2}\right)
\end{aligned}
$$

where

$$
\begin{aligned}
\mathrm{T}_{1} & =\left\{\mathrm{R} \overline{\mathrm{p}}_{3}^{2}-2 \mathrm{~B} / \varepsilon\left(\overline{\mathrm{q}}_{2}+\mathrm{A}_{\lambda}\right)\right\}\left(\mathrm{A}_{\lambda, \theta} \overline{\mathrm{q}}_{3}+\mathrm{A}_{\psi, \theta} \overline{\mathrm{p}}_{3}+\mathrm{A}_{\lambda} \mathrm{A}_{\psi, \theta}\right) \\
& +\left(\sqrt{ } \varepsilon\left(\overline{\mathrm{q}}_{2}+\mathrm{A}_{\lambda}\right)\right)^{2}\left(\mathrm{R}_{, \psi} \overline{\mathrm{p}}_{3}+\mathrm{R}, \lambda \overline{\mathrm{q}}_{3}+\mathrm{R}, \psi \mathrm{A}_{\lambda}-\mathrm{R}_{, \lambda} \mathrm{A}_{\psi}\right) \\
& +\left(\mathrm{E}_{, \psi} \mathrm{A}_{\lambda}-\mathrm{E}_{, \lambda} \mathrm{A}_{\psi}+\mathrm{E}_{, \psi}, \overline{\mathrm{P}}_{3}+\mathrm{E}_{, \lambda} \overline{\mathrm{q}}_{3}\right) \overline{\mathrm{q}}_{3}^{2}
\end{aligned}
$$




$$
\begin{aligned}
&+2\left(F_{, \psi} A_{, \lambda}-F_{, \lambda} A_{\psi}+F, \psi \bar{p}_{3}+F, \lambda \bar{q}_{3}\right) \bar{q}_{3} \bar{p}_{3} \\
&+\left(G_{, \psi} A_{\lambda}-G_{, \lambda} A_{\psi}+G_{, \psi} \bar{p}_{3}+G_{, \lambda} \bar{q}_{3}\right) p^{-2} \\
&+2 E \bar{q}_{3}\left(A_{\psi, \psi} A_{\lambda}-A_{\psi, \lambda} A_{\psi}+A_{\psi, \psi} \bar{p}_{3}+A_{\psi, \lambda} \bar{q}_{3}\right) \\
&+2 F\left\{-\bar{q}_{3}\left(A_{\lambda, \psi} A_{\lambda}-A_{\lambda, \lambda} A_{\psi}+A_{\lambda, \psi} \bar{p}_{3}+A_{\lambda, \lambda} \bar{q}_{3}\right)\right. \\
&\left.+\bar{p}_{3}\left(A_{\psi, \psi} A_{\lambda}-A_{\psi, \lambda} A_{\psi}+A_{\psi, \psi} \bar{p}_{3}+A_{\psi, \lambda} \bar{q}_{3}\right)\right\} \\
&+2 G \bar{p}_{3}\left(A_{\lambda, \psi} A_{\lambda}-A_{\lambda, \lambda} A_{\psi}+A_{\lambda, \psi} \bar{p}_{3}+A_{\lambda, \lambda} \bar{q}_{3}\right)
\end{aligned}
$$

and where the replacement of the functions of space $\psi, \lambda, \theta$, is $\psi=$ $\varepsilon^{3 / 2} \overline{\mathrm{p}}_{1}+\varepsilon \cdot \overline{\mathrm{q}}_{2}, \lambda=\varepsilon \overline{\mathrm{p}}_{2}$, and $\theta=\overline{\mathrm{q}}_{1}+\sqrt{ } \varepsilon \overline{\mathrm{p}}_{2}$.

We now start the construction of the lowest order magnetic moment adiabatic invariant. We see that through order $\sqrt{ } \varepsilon$ the curves $H=$ const. in the $\bar{n}_{3}, \bar{q}_{3}$ plane are slightly off-center ellipses. An affine transformaion will shift and stretch the ellipses to circles and so we introduce the next generator in terms of the as yet unspecified functions $\alpha(\psi, \lambda), \beta(\psi, \lambda), \gamma(\psi, \lambda)$, and $\delta(\psi, \lambda)$

$$
\begin{aligned}
F= & \left(\alpha \ddot{p}_{3}+v \varepsilon \gamma \sqrt{ } \varepsilon\left(\bar{q}_{2}+A_{\lambda}\right)\right) \cdot\left(\bar{q}_{3}+\sqrt{ } \delta \delta \sqrt{ } \varepsilon\left(\bar{q}_{2}+A_{\lambda}\right)\right) \\
& +\beta \tilde{p}_{3}^{2} / 2+\tilde{p}_{1} \bar{q}_{1}+\tilde{p}_{2} \bar{q}_{2}
\end{aligned}
$$

where

$$
\begin{aligned}
& \psi=\varepsilon^{3 / 2} \tilde{p}_{1}+\varepsilon \bar{q}_{2} \\
& \lambda=\varepsilon \tilde{p} \\
& \theta=\bar{q}_{1}+\gamma \varepsilon \tilde{p}_{2}
\end{aligned}
$$




$$
\begin{aligned}
& \bar{p}_{1}=\tilde{p}_{1}+o(\sqrt{ }), \quad \bar{q}_{1}=\tilde{q}_{1}+o\left(\varepsilon^{3 / 2}\right), \\
& \bar{p}_{2}=\tilde{p}_{2}+o(\sqrt{ } \varepsilon), \quad \bar{q}_{2}=\tilde{q}_{2}+o(\varepsilon) \\
& \bar{p}_{3}=\alpha \tilde{p}_{3}+\sqrt{\varepsilon} \gamma\left(\tilde{q}_{2}+A_{\lambda}\right) \\
& \bar{q}_{3}=-\beta \tilde{p}_{3} / \alpha+\tilde{q}_{3} / \alpha-\sqrt{ } \varepsilon \delta \sqrt{ } \varepsilon\left(\tilde{q}_{2}+A_{\lambda}\right),
\end{aligned}
$$

while the flux coordinate is essentially unchanged,

$$
\varepsilon^{3 / 2} \bar{p}_{1}+\varepsilon \bar{q}_{2}=\varepsilon^{3 / 2} \tilde{p}_{1}+\varepsilon \bar{q}_{2}+O\left(\varepsilon^{2}\right),
$$

with a time independent point function for error, which does not represent a drift. If we introduce the new variables into (A4) and set

$$
\begin{aligned}
& B^{2}=E G-F^{2} \\
& \alpha^{2}=E / B \\
& B=F / B \\
& \gamma=-R E / B^{2} \\
& s=-R E / B^{2}
\end{aligned}
$$

we find

$$
\begin{aligned}
H= & (1 / 2)\left(\tilde{p}_{3}^{2}+\tilde{\varphi}_{3}^{2}\right) B+(1 / 2) R\left(\sqrt{ } \varepsilon\left(\tilde{\varphi}_{2}+A_{\lambda}\right)\right)^{2} \cdot\left(1-\varepsilon k \dot{k}: / \mathrm{B}^{2}\right) \\
& +\left(\varepsilon \mathrm{T}_{1}\right) / 2+O\left(\varepsilon^{3 / 2}\right)
\end{aligned}
$$

where we make the substitutions

$$
\begin{aligned}
& \overline{\mathrm{p}}_{3}=\alpha \tilde{p}_{3} \\
& \bar{q}_{3}=-\beta \tilde{\mathrm{p}}_{3} / \alpha+\tilde{q}_{3} / \alpha
\end{aligned}
$$

in the expression (A5) for $\mathrm{T}_{1}$. Functions of space are now evaluated at $\psi=\varepsilon^{3 / 2} \tilde{p}_{1}+\varepsilon \tilde{q}_{2}, \lambda=\varepsilon \cdot \tilde{p}_{2}, \theta=\tilde{q}_{1}+\sqrt{ } \varepsilon \tilde{p}_{2}$.

As we need the Hamiltonian to first order in $\varepsilon$ we must get the 
magnetic moment correct through order $\varepsilon$. For clarity and simplicity let us consider the following problem in Hamiltonian dynamics in the canonically conjugate set of variables $(p, q)$,

$$
H=(1 / 2) A\left(p^{2}+q^{2}\right)+\varepsilon A(p, q),
$$

where $P(p, q)$ is a polynomial of degree $N$ in $p, q$. Suppose we introduce the generator of a transformation near the identity

$$
\mathrm{F}=\overline{\mathrm{p}} \mathrm{q}+\varepsilon Q(\overline{\mathrm{p}}, \mathrm{q}),
$$

where $Q(\bar{p}, q)$ is a polynomial of degree $N$ in $(\bar{p}, q)$, so that

$$
\begin{aligned}
& \bar{q}=q+\varepsilon \frac{\partial Q}{\partial \bar{p}} \\
& p=\bar{p}+\varepsilon \frac{\partial Q}{\partial q},
\end{aligned}
$$

or

$$
\begin{aligned}
& q=\bar{q}-\varepsilon \frac{\partial Q(\bar{p}, \bar{q})}{\partial \bar{p}}+O\left(\varepsilon^{2}\right) \\
& p=\bar{p}+\varepsilon \frac{\partial Q(\bar{p}, \bar{q})}{\partial \bar{q}}+O\left(\varepsilon^{2}\right)
\end{aligned}
$$

and the Hamiltonian becomes

$$
H=A \cdot\left(\bar{p}^{2}+\bar{q}^{2}\right) / 2+\varepsilon\left\{A\left(\bar{p} \frac{\partial Q \underline{Q}}{\partial \bar{q}}-\bar{q} \frac{\partial Q}{\partial \bar{p}}\right)+P(\bar{p}, \bar{q})\right\}+O\left(\varepsilon^{2}\right)
$$

We seek to select $Q(\bar{p}, \bar{q})$ so as to remove as much of the $O(\varepsilon)$ terms in $H$ as possible. We introduce polar soordinates in the $\bar{p}, \bar{q}$ plane such that $\overline{\mathrm{p}}=\rho \cos \theta, \overline{\mathrm{q}}=\rho \sin \theta$ and we find

$$
\left.A\left(\bar{p} \frac{\partial Q}{\partial \bar{q}}-\bar{q} \frac{\partial Q}{\partial \bar{p}}\right)+P(\bar{p}, \bar{q})=A \frac{\partial Q}{\partial \theta}+P(\rho \cos \theta, \rho \sin \theta)\right) \text {. Thus, if we }
$$


express $P(\rho \cos \theta, \rho \sin \theta)$ as a Fourier series in $\theta$, then by an appropriate choice of $Q$ we may eliminate all but the constant terms. Specifically if $P=\bar{p}^{m} \bar{q}^{n}$, then we may eliminate the term if $m+n$ is not even, while if $m+n=2 r$, then we may transform $\overline{\mathrm{p}}^{2 r-n} \overline{\mathrm{q}}^{\mathrm{n}}$ into $\left(\overline{\mathrm{p}}^{2}+\right.$ $\left.\bar{q}^{2}\right)^{r} \int_{0}^{2 \pi}(\cos \theta)^{2 r-n}(\sin \theta)^{n} d \theta / 2 \pi$. We now apply this procedure to our Hamiltonian and we extend the transformation to the full set of variablec. We readily find

$$
\begin{aligned}
H= & (1 / 2)\left(\tilde{p}_{3}^{2}+\tilde{q}_{3}^{2}\right)\left[B+\varepsilon\left\{R E+2 B^{3}\left(\left(A_{\psi} / B\right), \lambda-\left(A_{\lambda} / B\right), \psi,\right\} / 2 B\right]\right. \\
& +R\left(\sqrt{ } \varepsilon\left(\tilde{q}_{2}+A_{\lambda}\right)\right)^{2}\left(1-\varepsilon R E / B^{2}\right) \\
& -2 \varepsilon R / \varepsilon\left(\tilde{q}_{2}+A_{\lambda}\right) A_{\lambda} A_{\psi, \theta} \\
& +\varepsilon\left(\sqrt{ } \varepsilon\left(\tilde{q}_{2}+A_{\lambda}\right)\right)^{2}\left(R_{, \psi} A_{\lambda}-R_{, \lambda} A_{\psi}\right)+O\left(\varepsilon{ }^{3 / 2}\right) .
\end{aligned}
$$

We could, of course, repeat the process to higher order in $\varepsilon$ to conclude that $\tilde{p}_{3}^{2}+\tilde{q}_{3}^{2}$ is constant, say $2 \mu$, to high order. We could work with (Al6) but it simplifies the subsequent analysis to make two further transtormations.

Let $f(\theta)$ be an arbitrary continuous function of $\theta$, periodic of period $2 \pi$ and with zero average

$$
\langle f(\theta)\rangle=\frac{1}{2 \pi} \int_{0}^{2 \pi} f(\theta) d \theta=0 .
$$

It is desirable to introduce an indefinite integral of $f(\theta)$, say If, such that $\operatorname{If}(\theta)$ is periodic of period $2 \pi$ and $\langle$ If $\rangle=0$. Our integral operator I applied to $\exp (i n \theta), n \neq 0$ yields

$$
I \exp (\operatorname{in} \theta)=\{\exp (\operatorname{in} \theta)\} /(i n),
$$

or equivalently, 


$$
\operatorname{If}(\theta)=\oint_{0}^{\theta} d \theta^{\prime} f\left(\theta^{\prime}\right)+\int_{0}^{2 \pi} \theta^{\prime} d \theta^{\prime} f\left(\theta^{\prime}\right) /(2 \pi)
$$

and it is readily verified that If is periodic of period $2 \pi$, $\langle$ If $\rangle=0$, and $(d / d \theta)($ If $)=f(\theta)$.

In (Al6) we now repace $\tilde{\mathrm{p}}_{3}^{2}+\tilde{q}_{3}^{2}$ by $2 \mu$ and consider the formula to. define a Hamiltonian $H\left(\tilde{p}_{1}, \tilde{q}_{1}, \tilde{p}_{2}, \tilde{q}_{2}\right)$. If we introduce the generator

$$
F=\overline{\mathrm{p}}_{1} \tilde{\tilde{q}}_{1}+\overline{\mathrm{p}}_{2} \tilde{\mathrm{q}}_{2}+\mathrm{IA}_{\lambda}\left(\varepsilon^{3 / 2} \overline{\mathrm{p}}_{1}+\varepsilon \tilde{\mathrm{q}}_{2}, \varepsilon \overline{\mathrm{p}}_{2} \cdot \tilde{\mathrm{q}}_{1}+v \varepsilon \overline{\mathrm{p}}_{2}\right) / \sqrt{ } \varepsilon,
$$

where the variables $\left(\bar{p}_{1}, \bar{q}_{1}\right),\left(\bar{p}_{2}, \bar{q}_{2}\right)$ are not to be confused with thase defined by (Al) and (A2), then we find

$$
\begin{aligned}
& \tilde{p}_{1}=\bar{p}_{1}+A_{\lambda}\left(\varepsilon^{3 / 2} \bar{p}_{1}+\varepsilon \tilde{q}_{2}, \varepsilon \bar{p}_{2}, \tilde{q}_{1}+\sqrt{ } \varepsilon \bar{p}_{2}\right) / \sqrt{ } \varepsilon \\
& \bar{q}_{1}=\tilde{q}_{1}+\varepsilon I A_{\lambda, \psi}\left(\varepsilon^{3 / 2} \bar{p}_{1}+\varepsilon \tilde{q}_{2}, \varepsilon \bar{p}_{2}, \tilde{q}_{2}+\sqrt{ } \varepsilon p_{2}\right) \\
& \tilde{\mathrm{p}}_{2}=\overline{\mathrm{p}}_{2}+\gamma \varepsilon I A_{\lambda, \psi}\left(\varepsilon^{3 / 2} \overline{\mathrm{p}}_{1}+\varepsilon \tilde{\mathrm{q}}_{2}, \varepsilon, \overline{\mathrm{p}}_{2}, \tilde{\mathrm{q}}_{1}+\sqrt{ } \varepsilon \overline{\mathrm{p}}_{2}\right) \\
& \overline{\mathrm{q}}_{2}=\tilde{\mathrm{q}}_{2}+\mathrm{A}_{\lambda}\left(\varepsilon^{3 / 2} \overline{\underline{p}}_{1}+\varepsilon \tilde{\mathrm{q}}_{2}, \varepsilon \overline{\mathrm{p}}_{2}, \tilde{\mathrm{q}}_{1}+\sqrt{ }, \overline{\mathrm{p}}_{2}\right)
\end{aligned}
$$

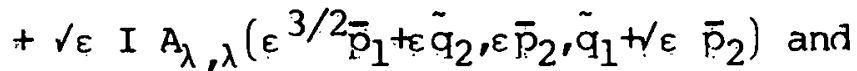

$$
\begin{aligned}
& H=\mu\{B+\varepsilon R E /(2 B)\}+\left(\sqrt{ } \varepsilon \bar{q}_{2}\right)^{2}\left(R-\varepsilon R^{2} E / B\right) / 2 \\
& +\varepsilon \mu B^{2}\left(\left(A_{\psi} / B\right), \lambda-\left(A_{\lambda} / B\right), \psi\right)-\varepsilon R A_{\lambda} A_{\psi, \theta}\left(\sqrt{ } \varepsilon \bar{q}_{2}\right) \\
& +\varepsilon\left(\sqrt{ } \varepsilon \bar{q}_{2}\right)^{2}\left(R_{, \psi} A_{\lambda}-R_{\lambda} A_{\psi}\right) / 2+o\left(\varepsilon^{3 / 2}\right) \text {, }
\end{aligned}
$$

and the flux coordinate is not changed in an essential way:

$$
\varepsilon^{3 / 2} \overline{\mathrm{p}}_{1}+\varepsilon \overline{\mathrm{q}}_{2}=\varepsilon^{3 / 2} \tilde{\mathrm{p}}_{1}+\varepsilon \tilde{\mathrm{q}}_{2}+o\left(\varepsilon^{3 / 2}\right),
$$

where the error is a time independent point function not representing a particle drift. We could easily carry this computation to higher order if needed or desịred.

one final transformation, akin to the treatment of simplifies the structure of the Hamiltonian (Al9) consinerabily. We let 
$f(\psi, \lambda, \theta)$ and $g(\psi, \lambda, \theta)$ be two arbitrary continuous functions, periodic in $\theta$ and with zero average and we introduce the generator

$$
F=\hat{p}_{1} \bar{q}_{1}+\hat{p}_{2} \bar{q}_{2}+I F+\sqrt{ } \varepsilon \bar{q}_{2} I g,
$$

where If and Ig are evaluated at $\psi, \lambda, \theta$ with $\psi=\varepsilon^{3 / 2} \hat{\mathrm{p}}_{1}+\varepsilon \bar{q}_{2}, \lambda=$ $\varepsilon \hat{\mathrm{p}}_{2}$ and $\theta=\overline{\mathrm{q}}_{1}+\sqrt{ } \varepsilon \hat{\mathrm{p}}_{2}$. If we set

$$
\begin{aligned}
& g=\left(R_{, \psi} A_{\lambda}-R,_{, \lambda} A_{\psi}\right) /(2 R) \\
& f=A_{\lambda} A_{\psi, \theta}-\left\langle A_{\lambda} A_{\psi, \theta}\right\rangle
\end{aligned}
$$

then

$$
\begin{aligned}
& \mathrm{H}\left(\varepsilon^{3 / 2} \hat{\mathrm{p}}_{1}+\varepsilon \hat{\mathrm{q}}_{1}, \varepsilon \hat{\mathrm{p}}_{2}, \sqrt{ } \varepsilon \hat{\mathrm{q}}_{2}, \hat{\mathrm{q}}_{1}+\sqrt{ } \hat{\mathrm{p}}_{2}\right) \\
& =\mu(\mathrm{B}+\varepsilon \mathrm{RE} /(2 \mathrm{~B}))+\left(\sqrt{\varepsilon} \hat{\mathrm{q}}_{2}\right)^{2}\left(\mathrm{R}-\varepsilon \mathrm{R}^{2} \mathrm{E} / \mathrm{B}\right) / 2 \\
& \quad-\varepsilon \mathrm{R}\left(\sqrt{ } \hat{\mathrm{q}}_{2}\right)\left\langle\mathrm{A}_{\lambda} \mathrm{A}_{\psi, \theta}>+\varepsilon \mu \mathrm{B}^{2}\left(\left(\mathrm{~A}_{\psi} / \mathrm{B}\right)_{, \lambda}-\left(\mathrm{A}_{\lambda} / \mathrm{B}\right)_{, \psi}\right)+\mathrm{O}\left(\varepsilon^{3 / 2}\right)\right. \text { where }
\end{aligned}
$$

functions of space are evaluated at $(\psi, \lambda, \theta)$ and

$$
\begin{aligned}
& \psi=\varepsilon \hat{\varepsilon}^{3 / 2} \hat{p}_{1}+\varepsilon \hat{\mathrm{q}}_{2} \\
& \lambda=\varepsilon \hat{\mathrm{p}}_{2} \\
& \theta=\hat{\mathrm{q}}_{1}+\sqrt{\varepsilon} \hat{\mathrm{p}}_{2} .
\end{aligned}
$$

The scaling for our variables is $\varepsilon^{3 / 2} \hat{p}_{1}, \varepsilon \hat{p}_{2}$ and $\sqrt{ } \varepsilon \hat{q}_{2}$ are all $O(1)$ and we do not need to scale. $\hat{q}_{1}$. Further, the flux coordinate of a particte is

$$
\psi=\varepsilon^{3 / 2} \hat{p}_{1}+\varepsilon \hat{q}_{2}+O\left(\varepsilon^{3 / 2},\right.
$$

where the error is independent of time and does not represent a particle drift, but rather change of coordinates. 
Acknowiedgements:

This work originated after extensive discussions after Dr. James A. Rome. His exhaustive numerical computations, both published and unpublished, demonstrated the phenomena this work set out to find. The aouthor is deeply grateful for his advice and comments. Discussions with Drs. Homer K. Meier and Alan Boozer are appreciated. Some of this work was done while the author was visiting: the Fusion Energy Division of Oak Ridge National Jaboratory and their hospitality and support are gratefully acknowledged. The work was supported: by the U. S. Department of Energy Contract DF-AC02-76ER03077 and the Air Force Office of Scientific Research contract No. 76-2881.

\section{Footnotes}

(1) The research monograph by T. G. Northrop, The Adiabatic Motion of Charged Particles (Wiley, New York 1963), contains essentially all the: older results and has a thorough biblioqraphy.

(2) See, for instance J. A. Rome and Y-K. M. Peng, Nucl. Fusion 19, 1193 (1979) and A. H. Boozer, Phys. Fluids 23, 904 (1980).

(3) C. S. Gardner, Phys. Rev. 115, 791 (1959).

(4) T. G. Northrop and J. A. Rome, Phys. Eluids 21, 384 (1978)..

(5) Private ommunication. A. H. Boozer, see also his work cited in note (2):

(6) E. T. Whittaker, A Treatise on the Analytical Dynamics of Particles and' Rigid Bodies, Fourth erition (Dover Publications, New York, 1944), Section 141 , p. $313^{\prime}$

(7) E. T. Whittaker, Ibid.., Section 131, p. 300. 
This report was prepared as an account of Government sponsored work. Neither the United States, nor the Administration, nor any person arting on behalf of the Administration:

A. Makce any warranty or represenlaliun, express or implied, with respect to the accuracy, completeness, or usefulness of the information contained in this report. or that the use of any. information, apparatus, method, or process disclosed in this report may not infringe privately owned rights; or

B. Assumes any liabilities with respect to the use of, or for damages resulting from the use of any information, apparatus, method, or process disclosed in this report.

As used in the above, "person acting on behalf of the Adninistration" includes any employee or contractor of the Administration, or employee of such contractor, to the extent that such employee or contractor of the Administration, or employee of surh contractor prepares, disseminates, or provides access to, any information pursuant to his. employment or contract with the Administration, or his employment with such contractor. 